\title{
PROJECTIVE HULLS AND THE PROJECTIVE GELFAND TRANSFORM*
}

\author{
F. REESE HARVEY ${ }^{\dagger}$ AND H. BLAINE LAWSON, JR. ${ }^{\ddagger}$
}

Dedicated, with affection and deep esteem, to the memory of S.-S. Chern.

\begin{abstract}
We introduce the notion of a projective hull for subsets of complex projective varieties parallel to the idea of a polynomial hull in affine varieties. With this concept, a generalization of J. Wermer's classical theorem on the hull of a curve in $\mathbf{C}^{n}$ is established in the projective setting. The projective hull is shown to have interesting properties and is related to various extremal functions and capacities in pluripotential theory. A main analytic result asserts that for any point $x$ in the projective hull $\widehat{K}$ of a compact set $K \subset \mathbf{P}^{n}$ there exists a positive current $T$ of bidimension $(1,1)$ with support in the closure of $\widehat{K}$ and a probability measure $\mu$ on $K$ with $d d^{c} T=\mu-\delta_{x}$. This result generalizes to any Kähler manifold and has strong consequences for the structure of $\widehat{K}$.

We also introduce the notion of a projective spectrum for Banach graded algebras parallel to the Gelfand spectrum of a Banach algebra. This projective spectrum has universal properties exactly like those in the Gelfand case. Moreover, the projective hull is shown to play a role (for graded algebras) completely analogous to that played by the polynomial hull in the study of finitely generated Banach algebras.

This paper gives foundations for generalizing many of the results on boundaries of varieties in $\mathbf{C}^{n}$ to general algebraic manifolds.
\end{abstract}

Key words. Polynomial hull, Gelfand Transformation, analytic varieties and their boundaries, Jensen measures, extremal functions, quasi-plurisubharmonic functions, pluripolar sets

AMS subject classifications. 32E99, 46J99, 14C99

1. Introduction. A beautiful classical theorem of John Wermer $\left[\mathrm{W}_{1}\right]$ states that the polynomial hull $\widehat{\gamma}_{\text {poly }}$ of a compact real analytic curve $\gamma \subset \mathbf{C}^{n}$, has the property that $\widehat{\gamma}_{\text {poly }}-\gamma$ is a 1-dimensional complex analytic subvariety of $\mathbf{C}^{n}-\gamma$. (Recall that the polynomial hull of $K \subset \subset \mathbf{C}^{n}$ is the set of points $x \in \mathbf{C}^{n}$ such that $|p(x)| \leq \sup _{K}|p|$ for all polynomials $p$.)

This paper was largely motivated by the question:

Does there exist an analogous result for curves in complex projective space $\mathbf{P}^{n}$ ?

To this end we introduce the notion of the projective hull of a compact set $K \subset \mathbf{P}^{n}$. It is defined to be the set $\widehat{K}$ of points $x \in \mathbf{P}^{n}$ for which there exists a constant $C=C_{x}$ such that

$$
\|\mathcal{P}(x)\| \leq C^{d} \sup _{K}\|\mathcal{P}\|
$$

for all holomorphic sections $\mathcal{P}$ of $\mathcal{O}_{\mathbf{P}^{n}}(d)$ and all $d>0$. Strong motivation for this definition comes from the fact (Prop. 2.3) that if $\gamma$ is the boundary of a one-dimensional

\footnotetext{
* Received October 3, 2005; accepted for publication February 22, 2006.

${ }^{\dagger}$ Department of Mathematics, Rice University, P. O. Box 1892, Houston, TX 77251, USA (harvey@math.rice.edu).

$\ddagger$ Mathematics Department, Stony Brook University, Stony Brook, NY 11794-3651, USA (blaine @math.sunysb.edu). Research partially supported by the NSF.
} 
complex analytic subvariety $V \subset \mathbf{P}^{n}$, then $V \subseteq \widehat{\gamma}$. Furthermore, for large classes of non-trivial examples it is shown in $\S 9$ that $V=\widehat{\gamma}$.

The projective hull strictly generalizes the concept of the polynomial hull in the following sense. Suppose $K \subset \subset \Omega=$ an affine open subset of $\mathbf{P}^{n}$. Then

$$
\widehat{K}_{\text {poly }, \Omega} \subseteq \widehat{K}, \quad \text { and } \quad \widehat{K} \subset \subset \Omega \Rightarrow \widehat{K}_{\text {poly }, \Omega}=\widehat{K}
$$

where $\widehat{K}_{\text {poly }, \Omega}$ is defined as above using the regular functions (polynomials) on $\Omega$. The second statement, which is non-trivial, is proved in $\S 12$. The projective hull also satisfies a Local Maximum Modulus Principle which states that for any $K \subset \mathbf{P}^{n}$ and any bounded domain $U$ in some affine open subset $\Omega$, one has that $\widehat{K} \cap U$ is contained in the $\Omega$-polynomial hull of its boundary. (See Theorem 12.8 or Theorem 4 below).

The projective hull is always subordinate to the Zariski hull - if $K \subset Z \subset \mathbf{P}^{n}$ where $Z$ is an algebraic subvariety, then $\widehat{K} \subset Z$. Moreover, if a real curve $\gamma \subset \mathbf{P}^{n}$ is contained in an irreducible algebraic curve $Z$, then $\widehat{\gamma}=Z$.

Note that for $x \in \widehat{K}$ the infimum of the set of constants $C$ for which (1.1) holds is again such a constant. This best constant function $C_{K}: \widehat{K} \longrightarrow \mathbf{R}^{+}$plays a basic role in the study of projective hulls. It is bounded iff $\widehat{K}$ is compact, and it appears repeatedly in many contexts. It is sometimes convenient to extend $C_{K}$ to all of $\mathbf{P}^{n}$ by setting $C_{K}(x)=\infty$ for points $x \notin \widehat{K}$.

It is natural to ask for an interpretation of the projective hull in homogeneous coordinates. Let $\pi: \mathbf{C}^{n+1}-\{0\} \longrightarrow \mathbf{P}^{n}$ be the standard projection and for $K \subset \mathbf{P}^{n}$ set $S(K)=\pi^{-1}(K) \cap S^{2 n+1}$. The polynomial hull of $S(K)$ in $\mathbf{C}^{n+1}$ is a compact subset which is a union of disks centered at the origin. In $\S 5$ we prove that

$$
\widehat{K}=\pi\left\{\widehat{S(X)}_{\text {poly }}-\{0\}\right\} .
$$

The best constant function $C_{K}=1 / \rho_{K}$ where $\rho_{K}(x)$ is the radius of the disk in $\widehat{S(X)}_{\text {poly }}$ above $x$.

Interestingly, projective hulls have already appeared in a somewhat hidden way in pluripotential theory. The closest connection is in the work of Guedj and Zeriahi [GZ] who (following Demailly) considered on a general Kähler manifold $(X, \omega)$ the notion of a quasi-plurisubharmonic function. This is a real-valued function $v$ on $X$ which satisfies $d d^{c} v+\omega \geq 0$. The set of these functions is denoted $\mathcal{P S H}_{\omega}(X)$ and for each compact subset $K \subset X$ there is an associated extremal function

$$
\Lambda_{K}(x) \equiv \sup \left\{v(x): v \in \mathcal{P} \mathcal{S H}_{\omega}(X) \text { and }\left.v\right|_{K} \leq 0\right\} .
$$

Arguments in [GZ] show that for $X-\mathbf{P}^{n}$ the best constant function, extended to be $\equiv \infty$ on $\mathbf{P}^{n}-\widehat{K}$, satisfies

$$
\Lambda_{K}=\log C_{K} .
$$

For compact sets $K$ contained in a standard affine coordinate chart $\mathbf{C}^{n} \subset \mathbf{P}^{n}$ condition (1.1) for $z \in \mathbf{C}^{n}$ is equivalent to the condition that there exists $C>0$ with

$$
|p(z)| \leq C^{d} \sup _{K}|p|
$$

for all polynomials $p$ of degree $\leq d$ and all $d$. In this setting the best constant function is related to the Siciak extremal function defined in terms of the Lelong class of subharmonic functions with logarithmic growth [Si]. In particular the best constant 
function is finite at exactly those points where the Siciak function is finite. This is discussed in $\S 6$.

In pluripotential theory it is often customary to regularize extremal functions to be upper semicontinuous. In the cases of interest here such regularization gives $\Lambda_{K}^{*} \equiv \infty$. One can think of our results as showing that in this situation, the set where $\Lambda_{K}<\infty$ (namely $\left.\widehat{K}\right)$ has interesting structure, and so also does $\left.\Lambda_{K}\right|_{\widehat{K}}$.

The condition $\Lambda_{K}^{*} \equiv \infty$ is equivalent to $K$ having Bedford-Taylor capacity zero [BT]. It is also equivalent to $K$ being pluripolar , i.e., locally contained in the $-\infty$-set of a non-constant plurisubharmonic function (See $\S 4$ ). This points out the relative subtlety of the projective hull, since there exist smooth curves in $\mathbf{P}^{2}$ which are not pluripolar $[\mathrm{DF}]$.

Another close tie between polynomial and projective hulls comes from the theory of commutative Banach algebras. In 1941 Gelfand showed that to every Banach algebra $A$ there is a canonically associated compact Hausdorff space $X_{A}$ and a continuous embedding of $A$ into the algebra $C\left(X_{A}\right)$ of continuous complex-valued functions on $X_{A}$. (See $[\mathrm{G}]$, [Ho] or $\left[\mathrm{AW}_{1}\right]$.) The space $X_{A}$ is universal for representations of $A$ in the continuous functions on compact Hausdorff spaces. The points of $X_{A}$ are exactly the representations onto $C(\mathrm{pt}) \cong \mathbf{C}$, i.e., the multiplicative linear functionals.

Suppose now that $K$ is a compact subset of $\mathbf{C}^{n}$ and let $A(K)$ denote the uniform closure of the polynomials in $C(K)$. Then there is a canonical homeomorphism

$$
X_{A(K)} \cong \widehat{K}_{\text {poly }}
$$

of the Gelfand spectrum with the polynomial hull of $K$. This engenders a natural correspondence between finitely generated Banach algebras and polynomially convex subsets of $\mathbf{C}^{n}$, and enables one to employ the theory of several complex variables in the study of such algebras.

Now there is a completely parallel story relating projective hulls to Banach graded algebras. This parallel mimics the relationship between the Spectrum of a ring and Proj of a graded ring in modern algebraic geometry. A Banach graded algebra is a commutative $\mathbf{Z}^{+}$-graded normed algebra $A_{*}=\bigoplus_{k \geq 0} A_{k}$ where each $A_{k}$ is a Banach space. Typical examples are given by: $A_{k}=\Gamma_{\text {hol }}\left(X, \mathcal{O}\left(\lambda^{k}\right)\right)$ with the sup-norm, where $\lambda$ is a holomorphic hermitian line bundle on a complex manifold $X$, or $A_{k}=$ $\Gamma_{\text {cont }}\left(K, \lambda^{k}\right)$ with the sup-norm, for a hermitian line bundle $\lambda$ on a compact Hausdorff space $K$. In either case, when $X=K=\mathrm{pt}$, we have $A_{*} \cong \mathbf{C}[t]$.

For any Banach graded algebra $A_{*}$ we construct a topological space $\mathcal{X}_{A_{*}}$, called the projective spectrum of $A_{*}$ as the space of continuous homomorphisms $A_{*} \rightarrow \mathbf{C}[t]$ divided by the $\mathbf{C}^{\times}$-action corresponding to $\operatorname{Aut}(\mathbf{C}[t])$. The space $\mathcal{X}_{A_{*}}$ carries a hermitian line bundle $\lambda$, and there is a natural embedding

$$
A_{*} \hookrightarrow \bigoplus_{k \geq 0} \Gamma\left(\mathcal{X}_{A_{*}}, \lambda^{k}\right)
$$

called the projective Gelfand transformation.

Suppose now that $K$ is a compact subset of $\mathbf{P}^{n}$, and let $A_{*}(K)$ denote the restriction to $K$ of the homogeneous coordinate ring of $\mathbf{P}^{n}$ (represented by homogeneous polynomials in homogeneous coordinates). Then there is a canonical homeomorphism

$$
\mathcal{X}_{A_{*}(K)} \cong \widehat{K}
$$


of the projective spectrum with the projective hull of $K$. This engenders a natural correspondence between finitely generated Banach graded algebras and projectively convex subsets of $\mathbf{P}^{n}$.

The principal analytic result in this paper is the establishement of Jensen measures for points in the projective hull. The theorem has a number of interesting corollaries. In particular, with a mild hypothesis it yields the projective analogue of Wermer's theorem.

Fix $\Lambda>0$ and denote by $\mathcal{P}_{1,1}(\Lambda)$ the set of positive currents of bidimension $(1,1)$ with mass $\leq \Lambda$. For a compact set $K \subset \mathbf{P}^{n}$ let $\mathcal{M}_{K}$ denote the probablitiy measures on $K$ and let $\widehat{K}(\Lambda)$ denote the set of points in $x \in \widehat{K}$ with $\Lambda_{K}(x) \leq \Lambda$.

Main Analytic Theorem. For a compact subset $K \subset \mathbf{P}^{n}$ the following are equivalent:

$$
\text { (A) } \quad x \in \widehat{K}(\Lambda)
$$

(B) There exist $T \in \mathcal{P}_{1,1}(\Lambda)$ and $\mu \in \mathcal{M}_{K}$ such that:

$$
\begin{aligned}
& \text { (i) } d d^{c} T=\mu-\delta_{x} \\
& \text { (ii) } \operatorname{supp}(T) \subset \widehat{K}^{-} \equiv \text { the closure of } \widehat{K}
\end{aligned}
$$

Note. This theorem was inspired by a result of Duval-Sibony [DS, Thm. 4.2] in the affine case, and our proof incorporates their Hahn-Banach technniques. However, much more is required. Our projective result is (necessarily) quantitative. Furthermore, one must work in this case to find a current $T$ with support in the closure of the projective hull.

It is tempting to apply [DS] directly by considering the set $S(K) \subset S^{2 n+1} \subset \mathbf{C}^{n+1}$ in homogeneous coordinates discussed above, and then push their positive $(1,1)$ current $T$ forward to $\mathbf{P}^{n}$ by the projection. However, there is nothing in [DS] that indicates how to construct $T$ so that $0 \notin \operatorname{supp}(T)$. Indeed in homogeneous coordinates, much of the subtlety of this subject takes place near the origin.

As a corollary of the Main Analytic Result one can show that for any $x \in \widehat{K}^{-}$ there are probability measures $\nu \in \mathcal{M}_{\widehat{K}^{-}}, \mu \in \mathcal{M}_{K}$ and a current $T \in \mathcal{P}_{1,1}$ with $d d^{c} T=\mu-\nu$ and $x \in \operatorname{supp}(T) \subset \widehat{K}^{-}$.

Note that a current $T_{x} \in \mathcal{P}_{1,1}$ of least mass with support in $\widehat{K}^{-}$and $d d^{c} T_{x}=$ $\mu-\delta_{x}$ satisfies

$$
M\left(T_{x}\right)=\Lambda_{K}(x)
$$

Fix a compact subset $K \subset \mathbf{P}^{n}$. One of the important consequences of the main theorem is the following.

Theorem 1. The set $\widehat{K}^{-}-K$ is 1-concave in $\mathbf{P}^{n}-K$.

One-concavity is a strong local condition which means essentially no local peak points under holomorphic maps to C. (The definition is given in $\S 12$.) It has the following immediate consequence.

Corollary 1. $\widehat{K}^{-}-K$ has locally positive Hausdorff 2-measure.

Using Theorem 1 combined with work of Dinh and Lawrence [DL] or Sibony [Sib, Thm. 17] we conclude the following. 
THEOREM 2. If the Hausdorff 2-measure of $\widehat{K}^{-}-K$ is locally finite on some open subset $U \subset \mathbf{P}^{n}-K$, then $\left(\widehat{K}^{-}-K\right) \cap U$ is a 1-dimensional complex analytic subvariety of $U$.

THEOREM 3. Suppose that $K \subset \subset \mathbf{C}^{n}$ and let $\widehat{K}_{0}$ be a connected component of $\widehat{K}^{-}-K$ which is bounded in $\mathbf{C}^{n}$. Then $\widehat{K}_{0}$ is contained in the polynomial hull of $K$.

Corollary 3. If $\widehat{K} \subset \subset \Omega=\mathbf{P}^{n}-D$ for some algebraic hypersurface $D$, then $\widehat{K}=\widehat{K}_{\Omega}$. In particular if $\gamma \subset \Omega$ is a $C^{1}$-curve and if $\widehat{\gamma} \subset \subset \Omega$, then $\widehat{\gamma}-\gamma$ is a 1-dimensional analytic subvariety of $\Omega-\gamma$.

One might conjecture that if $\widehat{\gamma}$ is not an algebraic subvariety, then it is contained in the complement of some divisor, and Corollary 2 would give a projective version of Wermer's Theorem. However, recent beautiful work of Bruno Fabre $\left[\mathrm{Fa}_{1}\right]$ shows that this is far from true. His results are discussed in $\S 8$.

Using our Main Theorem we establish the following local structure theorem which yields, in particular, the Local Maximum Modulus Property for projective hulls.

THEOREM 4. For any bounded domain $U \subset \subset \Omega^{\text {affine open }} \subset \mathbf{P}^{n}$,

$$
\widehat{K}^{-} \cap U \subseteq\{(\widehat{K} \cap \partial U) \cup(K \cap U)\}_{\mathrm{poly}, \Omega} .
$$

We also obtain the following generalization of Wermer's Theorem.

TheOREm 5. Let $\gamma \subset \mathbf{P}^{n}$ be a finite union of real analytic curves. Then $\widehat{\gamma}$ has Hausdorff dimension 2. Furthermore, if the Hausdorff 2-measure of $\widehat{\gamma}^{-}$is finite in a neighborhood of some algebraic hypersurface, then $\widehat{\gamma}-\gamma$ is a 1-dimensional complex analytic subvariety of $\mathbf{P}^{n}-\gamma$.

The same conlcusion holds for any smooth pluripolar curve $\gamma$ in $\mathbf{P}^{2}$.

Theorems 1-5 are proved in $\S 12$.

Added in Proof. John Wermer has recently adapted an argument of E. Bishop to show the following. Let $\gamma \subset \mathbf{P}^{2}$ be a finite union of real analytic curves and let $\pi: \mathbf{P}^{2}-\mathbf{P}^{0} \rightarrow \mathbf{P}^{1}$ be linear projection from a point $\mathbf{P}^{0} \notin \widehat{\gamma}$. Suppose $\widehat{\gamma}$ is compact. Then $\left.\pi\right|_{\widehat{\gamma}}$ is finitely sheeted almost everywhere over $\mathbf{P}^{1}$. Combined with Theorem 12.8 below and Theorem 11.2 in $\left[\mathrm{W}_{0}\right]$ one concludes the following.

THEOREM 5'. Let $\gamma \subset \mathbf{P}^{n}$ be a finite union of real analytic curves and suppose $\widehat{\gamma}$ is compact, i.e., suppose the best constant function $C_{\gamma}$ is bounded above. Then $\widehat{\gamma}-\gamma$ is a 1-dimensional complex analytic subvariety of $\mathbf{P}^{n}-\gamma$.

Given a complex manifold $X$ and a hermitian holomorphic line bundle $\lambda \rightarrow X$ there is an analogue of the projective hull of $K \subset \subset X$ defined to be the set $\widehat{K}_{\lambda}$ of points $x \in X$ satisfying (1.1) for all $\mathcal{P} \in H^{0}\left(X, \mathcal{O}\left(\lambda^{d}\right)\right)$ and all $d>0$. There is also an analogue $\Lambda_{K, \lambda}$ of the extremal function (1.2). This is discussed in $\S 17$ where we prove that the projective hull is intrinsic, namely:

TheOREM 6. Suppose that $X \subset \mathbf{P}^{N}$ is an algebraic manifold and $\lambda=\mathcal{O}_{X}(1)$. Then for any compact subset $K \subset X$ we have

$$
\Lambda_{K, \lambda}=\left.\Lambda_{K}\right|_{X} \quad \text { and } \quad \widehat{K}_{\lambda}=\widehat{K}
$$


The reader may recall that extremal functions can be defined on any Kähler manifold $(X, \omega)$ using the quasi-plurisubharmonic functions by (1.2). One can therefore define the $\omega$-hull $\widehat{K}$ of a compact subset $K \subset X$ to be the set of points $x \in X$ where $\Lambda_{K}(x)<\infty$.

In $\S 18$ we establish the following.

Main Analytic Theorem for Arbitrary Kähler Manifolds. Let $X$ be any Kähler manifold. Then for any compact subset $K \subset X$ with $\widehat{K} \subset \subset X$ the following are equivalent.

$$
\begin{aligned}
& \text { (A) } x \in \widehat{K}(\Lambda) \\
& \text { (B) There exist } T \in \mathcal{P}_{1,1}(X) \text { with } M(T) \leq \Lambda \text { and } \operatorname{supp}(T) \subset \widehat{K}^{-} \text {such that } \\
& \qquad d d^{c} T=\mu-\delta_{x}
\end{aligned}
$$

where $\mu \in \mathcal{M}_{K}$.

The proof of this result is more rounded and conceptual than the one given for the special case in $\S 11$. Most of the consequences of the special case cited above carry over to the general setting.

We point out that this paper lays the foundation for a new characterization of boundaries of subvarieties in a compact Kahler manifold $X$. For $X=\mathbf{P}^{n}$ this problem has been studied in [Do], $\left[\mathrm{DH}_{1,2}\right]$, and $\left[\mathrm{HL}_{3}\right]$ where such boundaries were characterized in terms of analytic transforms and non-linear moment conditions. However, using results in this paper, the authors have formulated a quite different characterization of the boundaries of positive holomorphic chains in terms of projective linking numbers $\left[\mathrm{HL}_{4}\right]$. This generalizes the work of Alexander and Wermer $\left[\mathrm{AW}_{2}\right],\left[\mathrm{W}_{2}\right]$ in $\mathbf{C}^{n}$. The results in $\left[\mathrm{HL}_{4}\right]$ also cover the case of a general Kähler manifold $X$.

The authors would like to particularly thank Eric Bedford for several very useful conversations relating to this article. We also thank Tien-Chong Dinh and Vincent Guedj for explaining their results which have played an important role here. We are indebted to Nessim Sibony, Vincent Guedj, Ahmed Zeriahi, and Tien-Cuong Dinh for numerous useful comments. The second author would like to thank the Institut Henri Poincare and in particular Gennadi Henkin and Nessim Sibony for their hospitality during the development of this work.

2. The Projective Hull of a subset of $\mathbf{P}^{n}$. Let $\mathcal{O}(d) \longrightarrow \mathbf{P}^{n}$ denote the holomorphic line bundle of Chern class $d$ over complex projective $n$-space. Note that any hermitian metric on $\mathcal{O}(-1)$ naturally induces a hermitian metric on $\mathcal{O}(d)$ for each $d \in \mathbf{Z}$. This family of metrics has the property that

$$
\left\|v^{\otimes d}\right\|=\|v\|^{d} \quad \text { for any } v \in \mathcal{O}\left(d_{0}\right) \text {, any } d_{0}
$$

and $|(v, w)|=\|v\| \cdot\|w\|$ for $v \in \mathcal{O}(d), w \in \mathcal{O}(-d)$ for any $d$. Fix any such family of metrics and consider a compact subset $K \subset \mathbf{P}^{n}$.

Definition 2.1. The projective hull of $K$ is the subset $\widehat{K}$ of points $x \in \mathbf{P}^{n}$ with the following property: There exists a constant $C$ (depending on $x$ ) such that

$$
\|\sigma(x)\| \leq C^{d} \sup _{K}\|\sigma\|
$$


for all $\sigma \in H^{0}\left(\mathbf{P}^{n} ; \mathcal{O}(d)\right)$ and all $d \geq 0$.

The infimum of all $C$ for which (2.2) holds will be called the best constant function and denoted by $C_{K}(x)$.

EXERCISE 2.2. The hull $\widehat{K}$ is independent of the choice of hermitian metric on $\mathcal{O}(-1)$

The following fact was a primary motivation for considering this concept.

Proposition 2.3. Let $V$ be a compact connected Riemann surface with boundary $d V \neq \emptyset$. Suppose $f: V \rightarrow \mathbf{P}^{n}$ is a holomorphic map which extends holomorphically across the boundary. Then

$$
f(V) \subseteq \widehat{f(d V)}
$$

Proof. By assumption $V \subset \subset \widetilde{V}$ for some connected non-compact Riemann surface $\widetilde{V}$ and $f$ extends holomorphically to $\widetilde{V}$. Since $\widetilde{V}$ is Stein, there is a holomorphic trivialization of the pull-back $f^{*} \mathcal{O}(1) \stackrel{\cong}{=} \widetilde{V} \times \mathbf{C}$ which yields trivializations

$$
f^{*} \mathcal{O}(d) \stackrel{\cong}{\rightrightarrows} \widetilde{V} \times \mathbf{C},
$$

for all $d \geq 1$. With respect to (2.4) the pull-back metric is of the form

$$
\|v\|=\lambda^{d}|v|
$$

for some smooth function $\lambda: \widetilde{V} \rightarrow \mathbf{R}^{+}$.

Fix $p \in V-d V$ and $\sigma \in H^{0}\left(\mathbf{P}^{n}, \mathcal{O}(d)\right)$. Let $\widetilde{\sigma}: \widetilde{V} \rightarrow \mathbf{C}$ be the holomorphic function corresponding to $f^{*} \sigma$ under (2.4). Then applying the maximum principle to the compact subdomain $V \subset \widetilde{V}$ gives

$$
\|\widetilde{\sigma}(p)\|=\lambda(p)^{d}|\widetilde{\sigma}(p)| \leq \lambda(p)^{d} \sup _{d V}|\widetilde{\sigma}| \leq\left(\frac{\lambda(p)}{\mu}\right)^{d} \sup _{d V}\|\widetilde{\sigma}\|, \quad \text { where } \mu \equiv \inf _{d V} \lambda
$$

as desired.

This proof actually establishes the following.

Proposition 2.4. The conclusion (2.3) holds for any map $f: V \rightarrow \mathbf{P}^{n}$, holomorphic on $V-d V$ and continuous on $V$, such that the pull-back $f^{*} \mathcal{O}(1)$ admits a trivialization which is holomorphic on $V-d V$ and continuous on $V$.

REMARK 2.5. Although the projective hull is independent of the metric chosen on $\mathcal{O}(-1)$ it is convenient to work with the standard metric defined as follows. Recall that

$$
\mathcal{O}(-1)=\left\{(\ell, v) \in \mathbf{P}^{n} \times \mathbf{C}^{n+1}: v \in \ell\right\}
$$

and projection to the second factor gives a map $\operatorname{pr}_{2}: \mathcal{O}(-1) \rightarrow \mathbf{C}^{n+1}$ which is an isomorphism outside the zero-section and collapses the zero-section to the origin (the blow-up of 0 ). The standard metric on $\mathcal{O}(-1)$ is the unique hermitian metric whose unit circle bundle corresponds to the unit sphere $S^{2 n+1} \subset \mathbf{C}^{n+1}$ under the map $\operatorname{pr}_{2}$. 
Recall that any section $\sigma \in H^{0}\left(\mathbf{P}^{n}, \mathcal{O}(d)\right)$ gives a function $\tilde{\sigma}: \mathcal{O}(-1) \rightarrow \mathbf{C}$ which of degree $d$ on each fibre and descends under $\mathrm{pr}_{2}$ to a homogeneous polynmial $\widetilde{\sigma}: \mathbf{C}^{n+1} \rightarrow \mathbf{C}$ of degree $d$. This gives the identification

$$
H^{0}\left(\mathbf{P}^{n}, \mathcal{O}(d)\right) \cong \mathbf{C}\left[Z_{0}, \ldots, Z_{n}\right]_{d}, \quad d \geq 0
$$

with the space of homogeneous polynomials of degree $d$ in homogeneous coordinates.

Given a polynomial $P \in \mathbf{C}\left[Z_{0}, \ldots, Z_{n}\right]_{d}$, its standard norm at $x=[Z] \in \mathbf{P}^{n}$, when considered as a section $\mathcal{P}$ of $\mathcal{O}(d)$, is

$$
\|\mathcal{P}(x)\|=\frac{|P(Z)|}{\|Z\|^{d}} .
$$

In particular, given a subset $K \subset \mathbf{P}^{n}$, let $S(K)=\left\{z \in S^{2 n+1}: \pi(z) \in K\right\}$ where $\pi: \mathbf{C}^{n+1}-\{0\} \rightarrow \mathbf{P}^{n}$ is the homogeneous coordinate map. Then

$$
\sup _{K}\|\mathcal{P}\|=\sup _{S(K)}|P|
$$

for $P, \mathcal{P}$ as above.

3. Elementary Properties. The projective hull has several nice features.

Proposition 3.1.

(i) If $K \subseteq Y$, then $\widehat{K} \subseteq \widehat{Y}$.

(ii) If $Y$ is an algebraic subvariety, then $\widehat{Y}=Y$.

(iii) For any $K \subset \mathbf{P}^{n}, \widehat{K}$ is contained in the Zariski hull of $K$.

Proof. Part (i) is clear and (ii) $\Rightarrow$ (iii). To prove (ii) it suffices to show that if $D \subset \mathbf{P}^{n}$ is an algebraic hypersurface and $Y \subset D$, then $\widehat{Y} \subset D$. Write $D$ as $D=$ $\operatorname{Div}(\sigma)$ for some $\sigma \in H^{0}\left(\mathbf{P}^{n}, \mathcal{O}(d)\right)$. Then, $\left.Y \subset D \quad \Leftrightarrow \quad \sigma\right|_{Y}=\left.0 \Rightarrow \sigma\right|_{\widehat{Y}}=0 \Leftrightarrow$ $\widehat{Y} \subset D$.

The next result says that taking projective hulls commutes with Veronese reembeddings.

Proposition 3.2. Let $\widehat{K}_{k}$ denote the set of points $x \in \mathbf{P}^{n}$ for which there exists $C=C(x)$ such that $\|\sigma(x)\| \leq C^{d} \sup _{K}\|\sigma\|$ for all $\sigma \in H^{0}\left(\mathbf{P}^{n}, \mathcal{O}(d k)\right)$ and all $d \geq 1$. Then

$$
\widehat{K}_{k}=\widehat{K} .
$$

Proof. Suppose $x \in \widehat{K}$ and let $C=C(x)$ be the constant given in Definition 2.1. Then $\|\sigma(x)\| \leq\left(C^{k}\right)^{d} \sup _{K}\|\sigma\|$ for all $\sigma \in H^{0}\left(\mathbf{P}^{n}, \mathcal{O}(d k)\right)$ and so $x \in \widehat{K}_{k}$.

On the other hand, suppose $x \in \widehat{K}_{k}$ and let $C=C(x)$ be the constant in the definition above. Suppose $\sigma \in H^{0}\left(\mathbf{P}^{n}, \mathcal{O}(d)\right)$ is given. Then $\sigma(x)^{\otimes k} \in H^{0}\left(\mathbf{P}^{n}, \mathcal{O}(d k)\right)$ and so

$$
\|\sigma(x)\|^{k}=\left\|\sigma(x)^{\otimes k}\right\| \leq C^{d} \sup _{K}\left\|\sigma^{\otimes k}\right\|=C^{d} \sup _{K}\|\sigma\|^{k}=C_{0}^{d k}\left\{\sup _{K}\|\sigma\|\right\}^{k}
$$

where $C_{0} \equiv C^{1 / k}$. Taking $k$ th roots shows that $x \in \widehat{K}$. 
Recall that for any complex manifold $\Omega$ and any subset $K \subset \Omega$, the holomorphic hull of $K$ in $\Omega$ is the set

$$
\mathcal{H}_{\Omega}(K) \equiv\left\{x \in \Omega:|f(x)| \leq \sup _{K}|f| \text { for all } f \in \mathcal{O}_{K}\right\}
$$

Proposition 3.3. Let $\Omega \subset \mathbf{P}^{n}$ be any open subset containing $K$ with the property that Image $\left\{H^{1}\left(\mathbf{P}^{n}, \mathcal{O}^{\times}\right) \rightarrow H^{1}\left(\Omega, \mathcal{O}^{\times}\right)\right\}$is finite. Then

$$
\mathcal{H}_{\Omega}(K) \subset \widehat{K} .
$$

Corollary 3.4 .

$$
\bigcup_{\Omega=\mathbf{P}^{n}-D} \mathcal{H}_{\Omega}(K) \subseteq \widehat{K}
$$

where $D$ ranges over all divisors in $\mathbf{P}^{n}$.

Proof that $3.3 \Rightarrow$ 3.4. Image $\left\{H^{1}\left(\mathbf{P}^{n}, \mathcal{O}^{\times}\right) \rightarrow H^{1}\left(\mathbf{P}^{n}-D, \mathcal{O}^{\times}\right)\right\} \cong \mathbf{Z} / k$ where $k=\operatorname{degree}(D)$.

Proof of 3.3. By assumption there is an integer $k>0$ such that $\left.\mathcal{O}(k)\right|_{\Omega}$ is trivial. The argument given for Proposition 2.3 applies directly to prove that $\mathcal{H}_{\Omega}(K) \subseteq \widehat{K}_{k}=$ $\widehat{K}$.

REMARK 3.5. It should be noted that the containment in Corollary 3.4 is not an equality in general, even if one assumes that $K$ is a smoothly embedded $S^{1}$. See $\S 8$.

4. The Best Constant Function, Quasi-plurisubharmonicity and Pluripolarity. Definition 2.1 leads naturally to considering the family $\mathcal{S}_{K}$ of functions:

$$
\varphi=\frac{1}{d} \log \|\mathcal{P}\|, \quad \mathcal{P} \in H^{0}\left(\mathbf{P}^{n}, \mathcal{O}(d)\right) \text { for } d>0
$$

with the property that

$$
\varphi \leq 0 \quad \text { on } K \text {. }
$$

The associated extremal function

$$
\Lambda_{K}(x) \equiv \sup _{\varphi \in \mathcal{S}_{K}} \varphi(x)
$$

is the log of the best constant

$$
C_{K}(x)=\exp \left(\Lambda_{K}(x)\right)
$$

satisying (2.2). In particular

$$
\widehat{K}=\left\{x \in \mathbf{P}^{n}: \Lambda_{K}(x)<\infty\right\} .
$$

Each function $\varphi=\frac{1}{d} \log \|\mathcal{P}\| \in \mathcal{S}_{K}$ satisfies the current equation:

$$
d d^{c} \varphi=\operatorname{Div}(\mathcal{P})-\omega \quad \text { on } \mathbf{P}^{n}
$$

where the (1,1)-form $\omega$ is the standard Kähler form on $\mathbf{P}^{n}$. These important functions sit in the following, much larger, convex cone introduced by Demailly. 
Definition 4.1. A function $\varphi \in L^{1}\left(\mathbf{P}^{n}\right)$ is called quasi-plurisubharmonic (or $\omega$-quasi-plurisubharmonic) if it is an upper-semicontinuous, $[-\infty, \infty)$-valued function which satisfies

$$
d d^{c} \varphi+\omega \geq 0 \quad \text { on } \mathbf{P}^{n} .
$$

The set of these functions will be denoted by $\mathcal{P S H}_{\omega}$.

Note that $\mathcal{P} \mathcal{S H}_{\omega}$ contains $C^{\infty}$-functions as well as the highly singular ones in (4.1).

The following useful result can be found in [GZ, Proof of Thm. 4.2]. Analogues for general Kähler manifolds follow from work of Demailly $\left[\mathrm{D}_{*}\right]$.

Proposition 4.2. For any compact subset $K \subset \mathbf{P}^{n}$

$$
\Lambda_{K}(x) \equiv \sup \left\{\varphi(x): \varphi \in \mathcal{P} \mathcal{S H}_{\omega} \text { and }\left.\varphi\right|_{K} \leq 0\right\}
$$

Recall that a Borel subset $K \subset \mathbf{P}^{n}$ is called globally $\omega$-pluripolar if $K \subseteq$ $\left\{x \in \mathbf{P}^{n}: \varphi(x)=-\infty\right\}$ for some quasi-plurisubharmonic function $\varphi \leq 0$ which is not identically $-\infty$ on $\mathbf{P}^{n}$. The set $K$ is called (locally) pluripolar if every point $x \in K$ has a connected neighborhood $\mathcal{O}$ such that $K \cap \mathcal{O} \subseteq\left\{x \in \mathbf{P}^{n}: \varphi(x)=-\infty\right\}$ for some plurisubharmonic function $\varphi$ on $\mathcal{O}$ which is not identically $-\infty$.

Guedj and Zeriahi introduced and systematically studied quasi-plurisubharmonic functions in [GZ]. They also considered a notion of $\omega$-capacity, due originally to DinhSibony [DiS], and related it to capacities of Bedford-Taylor [BT], Alexander $\left[\mathrm{A}_{2}\right]$, Sibony-Wong $[\mathrm{SW}]$ and others. In all cases the sets of capacity zero are shown to be the same, and the following is proved.

Theorem 4.3. (Guedj-Zeriahi [GZ]). Let $K \subset \mathbf{P}^{n}$ be a compact subset, and denote by $\Lambda_{K}^{*}$ the upper-semicontinuous regularization of the function $\Lambda_{K}$. Then the following are equivalent:

(1) $\sup _{\mathbf{P}^{n}} \Lambda_{K}=\infty$.

(2) $\Lambda_{K}^{*} \equiv \infty$.

(3) $K$ has capacity zero.

(4) $K$ is globally $\omega$-pluripolar.

(5) $K$ is pluripolar.

Corollary 4.4. For a compact subset $K \subset \mathbf{P}^{n}$

$$
K \text { is pluripolar } \quad \Leftrightarrow \quad \widehat{K} \text { is pluripolar } \quad \Leftrightarrow \quad \widehat{K} \neq \mathbf{P}^{n} .
$$

Proof. If $K$ is pluripolar, then by Theorem 4.3(4) $K$ is contained in the $-\infty$ locus of a negative quasi-plurisubharmonic function $\varphi \not \equiv-\infty$ on $\mathbf{P}^{n}$. Now by (4.3) and Proposition 4.2, $x \in \widehat{K}$ iff $\varphi(x) \leq \sup _{K} \varphi+\Lambda_{K}(x)$ for all quasi-plurisubharmonic functions $\varphi$ on $\mathbf{P}^{n}$. Hence, $\varphi \equiv-\infty$ on $\widehat{K}$ and so $\widehat{K}$ is $\omega$-pluripolar. The converse is obvious, and the first equivalence is established.

Evidently if $\widehat{K}$ is pluripolar, then $\widehat{K} \neq \mathbf{P}^{n}$. However, if $\widehat{K} \neq \mathbf{P}^{n}$, then by definition $\Lambda_{K}$ is unbounded on $\mathbf{P}^{n}$ and hence $K$ is pluripolar by Theorem 4.3. 
REMARK 4.5. This corollary highlights the delicate nature of the projective hull. It is known (cf. $[\mathrm{DF}],[\mathrm{LMP}])$ that:

There exist $C^{\infty}$ closed curves in $\mathbf{P}^{2}$ which are not pluripolar.

The example in [LMP] actually bounds a holomorphic disk in $\mathbf{C}^{2}$. For this curve, the polynomial hull is the holomorphic disk and the projective hull is all of $\mathbf{P}^{2}$.

Note however that any real analytic curve is pluripolar, and therefore its projective hull is a proper subset of $\mathbf{P}^{n}$. A nice characterization of smooth graphs over the circle which are pluripolar is given in [CLP].

Note 4.6. A version of Theorem 4.3 is established in [GZ] with $\mathbf{P}^{n}$ replaced by any compact Kähler manifold (cf. §18).

Definition 4.7. By the pluripolar hull of a Borel set $K \subset \mathbf{P}^{n}$ we mean the set $\widehat{K}_{\mathrm{pp}}$ of points $x \in \mathbf{P}^{n}$ with the property that $\varphi(x)=-\infty$ for every non-constant $\varphi \in \mathcal{P} \mathcal{S H}_{\omega}$ with $\left.\varphi\right|_{K} \equiv-\infty$.

Proposition 4.8. For any compact subset $K \subset \mathbf{P}^{n}$, one has $\widehat{K} \subseteq \widehat{K}_{\mathrm{pp}}$.

Proof. Suppose $K \subset \varphi^{-1}(-\infty)$ for a non-constant $\varphi \in \mathcal{P S H}_{\omega}$. Then for every $c \in \mathbf{R}, \varphi+c \leq 0$ on $K$, and so $\varphi(x)+c \leq \Lambda_{K}(x)<\infty$. Hence, $\varphi(x) \leq \Lambda_{K}(x)-c$ for all $c \in \mathbf{R}$.

5. The Picture in Homogeneous Coordinates. Consider homogeneous cooordinates $\pi: \mathbf{C}^{n+1}-\{0\} \rightarrow \mathbf{P}^{n}$ and endow $\mathbf{C}^{n+1}$ with the standard hermitian metric. For any subset $K \subset \mathbf{P}^{n}$ set

$$
S(K) \equiv \pi^{-1}(K) \cap S^{2 n+1}
$$

where $S^{2 n+1} \subset \mathbf{C}^{n+1}$ is the unit sphere. Note that $S(K)$ is $S^{1}$-invariant, where $S^{1} \subset \mathbf{C}$ acts by scalar multiplication. In this section we shall characterize the polynomial hull $\widehat{K}$ in terms of $S(K)$.

Recall (2.7) that: $\|\mathcal{P}(x)\|=|P(Z)| /\|Z\|^{d}$ if $Z \in \mathbf{C}^{n+1}-\{0\}$ and $x=\pi Z$, where $\mathcal{P}$ is the section of $\mathcal{O}(d)$ corresponding to the homogeneous polynomial $P \in \mathbf{C}[Z]_{d}$ of degree $d$. Fix $x \in \mathbf{P}^{n}$. By definition $x \in \widehat{K}$ if and only if

$$
\|\mathcal{P}(x)\| \leq C^{d} \sup _{K}\|\mathcal{P}\|
$$

for all $\mathcal{P} \in H^{0}\left(\mathbf{P}^{n}, \mathcal{P}(d)\right)$ and $d \geq 0$. This condition can be restated in homogeneous coordinates as

$$
|P(Z)| \leq \sup _{S(K)}|P|
$$

for all $P \in \mathbf{C}[Z]_{d}, d \geq 0$ and for all $Z \in \mathbf{C}^{n+1}$ with $\pi(Z)=x$ and $\|Z\| \leq 1 / C$.

To see that (5.1) and (5.2) are equivalent recall that $\sup _{K}\|\mathcal{P}\|=\sup _{S(K)}|P|$ by (2.8). Substituting into (5.1) yields

$$
\frac{|P(Z)|}{\|Z\|^{d}} \leq C^{d} \sup _{S(K)}|P| .
$$

Hence, (5.1) implies (5.2). Now (5.2), with $Z \in \pi^{-1} x$ chosen so that $\|Z\|=1 / C$, is exactly (5.3), which implies (5.1) 
DEFINITION 5.1. The homogeneous polynomial hull of a subset $K \subset \mathbf{C}^{n+1}$ is the set $\widehat{K}_{\text {hom-poly }}$ of points $Z \in \mathbf{C}^{n+1}$ with the property that

$$
|P(Z)| \leq \sup _{K}|P|
$$

for all homogeneous polynomials $P$.

Given $K \subset \mathbf{P}^{n}$, let $\rho: \widehat{K} \rightarrow \mathbf{R}^{+}$be defined by

$$
\rho(x) \equiv \sup \left\{\|Z\|: \pi(Z)=x \quad \text { and } \quad Z \in \widehat{S(K)}_{\text {hom-poly }}\right\} .
$$

that is, the radius of the largest disk about zero in the line $\pi^{-1}(x)$ which is contained in the homogeneous polynomial hull of $S(K)$.

Proposition 5.2. For $K \subset \mathbf{P}^{n}$

$$
\rho(x)=\frac{1}{C_{K}(x)} \quad \text { for all } x \in \widehat{K}
$$

where $C_{K}$ is the best constant function (cf. (2.1)).

Proof. This is immediate from the equivalence of conditions (5.1) and (5.2) above.

Corollary 5.3. For any subset $K \subset \mathbf{P}^{n}$

$$
\widehat{K}=\pi\left\{\widehat{S(K)}_{\text {hom-poly }}-\{0\}\right\} .
$$

The following result is classical (cf. $\left.\left[\mathrm{A}_{2}\right]\right)$. We include a proof for completeness.

Proposition 5.4. For any $S^{1}$-invariant subset $K \subset \mathbf{C}^{n+1}$

$$
\widehat{K}_{\text {hom-poly }}=\widehat{K}_{\text {poly }}
$$

where $\widehat{K}_{\text {poly }}$ denotes the ordinary polynomial hull of $K$.

Proof. Clearly $\widehat{K}_{\text {poly }} \subseteq \widehat{K}_{\text {hom-poly }}$, and we need only prove $\widehat{K}_{\text {hom-poly }} \subseteq \widehat{K}_{\text {poly }}$. For this we use the following Lemma.

Lemma 5.5. Let $P$ be a polynomial in $\mathbf{C}^{n+1}$ and write $P=\sum_{m=0}^{N} P_{m}$ where $P_{m}$ is homogeneous of degree $m$. Then for any $Z \in \mathbf{C}^{n+1}$

$$
\left|P_{m}(Z)\right| \leq \sup _{\theta}\left|P\left(e^{i \theta} Z\right)\right|
$$

Proof. Note that $P(\lambda Z)=\sum_{m=0}^{N} \lambda^{m} P_{m}(Z)$, for $\lambda \in \mathbf{C}$, and therefore

$$
P_{m}(Z)=\left.\frac{1}{m !} \frac{\partial^{m}}{\partial \lambda^{m}} P(\lambda Z)\right|_{\lambda=0}=\frac{1}{2 \pi i} \int_{|\lambda|=1} \frac{P(\lambda Z) d \lambda}{\lambda^{m+1}}
$$

which gives that

$$
\left|P_{m}(Z)\right| \leq \frac{1}{2 \pi} \int_{0}^{2 \pi}\left|P\left(e^{i \theta} Z\right)\right| d \theta \leq \sup _{\theta}\left|P\left(e^{i \theta} Z\right)\right| .
$$


Corollary 5.6. Let $P=\sum_{m=0}^{N} P_{m}$ be as in Lemma 5.5, and suppose $K \subset \mathbf{C}^{n+1}$ is $S^{1}$-invariant. Then for $0 \leq m \leq N$ one has

$$
\sup _{K}\left|P_{m}\right| \leq \sup _{K}|P| \text {. }
$$

Suppose now that $Z \in \widehat{K}_{\text {hom-poly }}$ and let $P=\sum_{m=0}^{N} P_{m}$ be any polynomial decomposed as above. Then

$$
|P(Z)| \leq \sum_{m=0}^{N}\left|P_{m}(Z)\right| \leq \sum_{m=0}^{N} \sup _{K}\left|P_{m}\right| \leq \sum_{m=0}^{N} \sup _{K}|P|=(N+1) \sup _{K}|P| .
$$

Applying this to $P^{q}$ gives

$$
|P(Z)|^{q} \leq(N+1) q \sup _{K}|P|^{q}=(N+1) q\left(\sup _{K}|P|\right)^{q}
$$

and therefore

$$
|P(Z)| \leq(N q+q)^{\frac{1}{q}} \sup _{K}|P| .
$$

Since $\lim _{q \rightarrow \infty}(N q+q)^{\frac{1}{q}}=1$, we have $|P(Z)| \leq \sup _{K}|P|$, and so $Z \in \widehat{K}_{\text {poly }}$.

Combining Corollary 5.3 and Proposition 5.4 gives the following. Then

Theorem 5.7. For any subset $K \subset \mathbf{P}^{n}$ let $\widehat{K} \subset \mathbf{P}^{n}$ denote its projective hull.

$$
\widehat{K}=\pi\left\{\widehat{S(K)}_{\text {poly }}-\{0\}\right\} .
$$

6. The Affine Picture. Let $K \subset \mathbf{P}^{n}$ be a compact subset contained in an affine open chart $\mathbf{C}^{n}=\mathbf{P}^{n}-\mathbf{P}^{n-1}$.

Proposition 6.1. A point $z \in \mathbf{C}^{n}$ lies in $\widehat{K}$ if and only if there exists a constant $c>0$ such that

$$
|p(z)| \leq c^{d} \sup _{K}|p|
$$

for all polynomials $p \in \mathbf{C}\left[z_{1}, \ldots, z_{n}\right]$ of degree $\leq d$.

Proof. Choose homogeneous coordinates $\left[Z_{0}: \cdots: Z_{n}\right]$ for $\mathbf{P}^{n}$ such that $\mathbf{P}^{n-1}=$ $\left\{Z_{0}=0\right\}$. Choosing affine coordinates $\left(z_{1}, \ldots, z_{n}\right) \mapsto\left[1: z_{1}: \cdots: z_{n}\right]$ we identify $H^{0}\left(\mathbf{P}^{n}, \mathcal{O}(d)\right)$ with the space $\mathbf{C}\left[z_{1}, \ldots, z_{n}\right]_{\leq d}$ of polynomials of degree $\leq d$ (cf. (2.6)). (This results from the trivialization of $\mathcal{O}(d)$ over $\mathbf{C}^{n}$ via the section $Z_{0}^{d}$.) In this picture the standard metric has the form

$$
\|\mathcal{P}\|_{z}=\frac{|p(z)|}{\left(1+\|z\|^{2}\right)^{\frac{d}{2}}},
$$

where $\mathcal{P}$ denotes the section corresponding to $p$. Consequently a point $z \in \mathbf{C}^{n}$ lies in $\widehat{K}$ iff there is a constant $C$ such that

$$
\frac{|p(z)|}{\left(1+\|z\|^{2}\right)^{\frac{d}{2}}} \leq C^{d} \sup _{\zeta \in K}\left\{\frac{|p(\zeta)|}{\left(1+\|\zeta\|^{2}\right)^{\frac{d}{2}}}\right\}
$$


from which the result follows directly. by

In affine coordinates the family of functions $\mathcal{S}_{K}$ defined by (4.1) and (4.2) is given

$$
\varphi=\frac{1}{d} \log |p(z)|-\log \sqrt{1+|z|^{2}}
$$

where $p \in \mathbf{C}\left[z_{1}, \ldots, z_{n}\right]_{\leq d}$, with the property that

$$
\varphi \leq 0 \quad \text { on } K \subset \mathbf{C}^{n} .
$$

Proposition 6.1 suggests we consider the family $\mathcal{S}_{K}^{0}$ of plurisubharmonic functions

$$
\psi(z)=\frac{1}{d} \log |p(z)|
$$

for $p \in \mathbf{C}\left[z_{1}, \ldots, z_{n}\right]_{\leq d}$, with the property that

$$
\psi \leq 0 \quad \text { on } K
$$

and the associated extremal function

$$
\Lambda_{K}^{0}(z) \equiv \sup _{\psi \in \mathcal{S}_{K}^{0}} \psi(z)
$$

on $\mathbf{C}^{n}$. Note that for $x \in \mathbf{C}^{n}$,

$$
\Lambda_{K}^{0}(x)<\infty \quad \text { iff } \quad \Lambda_{K}(x)<\infty .
$$

Therefore, from (4.4) we have that for compact subsets $K \subset \mathbf{C}^{n}$,

$$
\widehat{K} \cap \mathbf{C}^{n}=\left\{z \in \mathbf{C}^{n}: \Lambda_{K}^{0}(z)<\infty\right\} .
$$

It is natural to expand $\mathcal{S}_{K}^{0}$ by using the Lelong class $\mathcal{L}$ of all plurisubharmonic functions $\psi$ on $\mathbf{C}^{n}$ such that $\psi(z) \leq c+\log (1+|z|)$ for some constant $c$. Set

$$
\mathcal{L}_{K} \equiv\{\psi \in \mathcal{L}: \psi \leq 0 \text { on } K\}
$$

In analogy with Proposition 4.2 one has

Proposition 6.2.

$$
\Lambda_{K}^{0}(z)=\sup _{\psi \in \mathcal{L}_{K}} \psi(z) .
$$

7. Theorems of Sadullaev. The extremal function $\Lambda_{K}^{0}$ was studied by A. Sadullaev who proved the following deep and beautiful result.

THEOREM 7.1 ([S]). Let $K \subset Z$ be a compact subset of an analytic subvariety $Z$ defined in some open subset of $\mathbf{C}^{n}$. Assume that $K$ is not pluripolar in $Z$. Then if $\Lambda_{K}^{0}$ (or equivalently $\Lambda_{K}$ ) is locally bounded on $Z$, the variety $Z$ must be algebraic.

Note. A subset $K \subset Z$ is pluripolar in $Z$ if for each point $x \in Z$ there is a neighborhood $\mathcal{O}$ of $x$ in $Z$ and a plurisubharmonic function $u: \mathcal{O} \rightarrow \mathbf{R}$, not identically $-\infty$, such that $K \cap \mathcal{O} \subseteq\{x \in \mathcal{O}: u(x)=-\infty\}$.

Sadullaev also proved the following.

Theorem $7.2([\mathrm{~S}])$. Let $A \subset \mathbf{C}^{n}$ be an irreducible algebraic curve, and $K \subset A$ a compact subset which is not pluripolar (equivalently, has positive capacity) in $A$. Then the extremal function $\Lambda_{K}^{0}$ is harmonic on $A-K$. 
More generally if $A$ is an algebraic subvariety of dimension $m$, then $\Lambda_{K}^{0}$ is the limit of an increasing sequence of maximal functions on $A-K$ (cf. [BT]).

We adapt the arguments of [S] to prove the following useful result.

THEOREM 7.3. Let $Z$ be a regular complex analytic curve defined in some open subset of $\mathbf{C}^{n}$ and consider a compact subset $K \subset Z$. Suppose $\mathcal{O} \subset \mathbf{C}^{n}-K$ is an open set with the property that

$$
\Lambda_{K}^{0} \equiv \infty \quad \text { in } \quad \mathcal{O}-Z
$$

Then in every connected component of $\mathcal{O} \cap Z$ either $\Lambda_{K}^{0} \equiv \infty$ or $\Lambda_{K}^{0}$ is a bounded harmonic function.

In particular if $\Lambda_{K}^{0} \equiv \infty$ in $\sim Z$, then in every connected component of $Z-K$ either $\Lambda_{K}^{0} \equiv \infty$ or $\Lambda_{K}^{0}$ is a bounded harmonic function.

Proof. Choose analytic coordinates $\left(\zeta, z_{1}, \ldots, z_{n-1}\right)$ for $|\zeta|<3$ and $\|z\|<1$ on a subset $\mathcal{O}_{0}$ of $\mathcal{O}$ such that $\mathcal{O}_{0} \cap Z$ is defined by $z=0$. Let $D=\{(\zeta, 0):|\zeta| \leq 1\}$.

Recall from Proposition 6.2 that $\Lambda_{K}^{0}(x)=\sup \left\{u(x): u \in \mathcal{L}_{K}\right\}$.

Lemma 7.4. Fix $u \in \mathcal{L}_{K}$ and consider the function $v: Z \rightarrow \mathbf{R}$ such that $v=u$ in $Z-D$ and $v$ is the harmonic extension of $\left.u\right|_{\partial D}$ to $D$. Then for every $\epsilon>0$ there exists a function $u_{\epsilon} \in \mathcal{L}_{K}$ whose restriction to $Z$ satisfies $u_{\epsilon} \geq \max \{u, v-\epsilon\}$.

Proof. Let $\pi(\zeta, z)=\zeta$ be projection in the coordinate bidisk, and set $\widetilde{v}=v \circ \pi$. Choose $\rho>0$ sufficiently small that

$$
\widetilde{v}-\epsilon<u \quad \text { on the set }\{(\zeta, z):|\zeta|=2 \text { and }\|z\| \leq \rho\} .
$$

Since $\Lambda_{K}^{0}(\zeta, z)=\infty$ for $\zeta \neq 0$, a standard compactness argument shows that for any $\gamma \in \mathbf{R}$ there exists a finite set of functions $\varphi_{1}, \ldots, \varphi_{N} \in \mathcal{L}_{K}$ such that $\varphi \equiv$ $\max \left\{\varphi_{1}, \ldots, \varphi_{N}\right\}>\gamma$ for $|\zeta| \leq 2$ and $\|z\|=\rho$. In particular we may assume that

$$
\widetilde{v}<\varphi \quad \text { on the set }\{(\zeta, z):|\zeta| \leq 2 \text { and }\|z\|=\rho\} .
$$

We now define

$$
u_{\epsilon} \equiv \begin{cases}\max \{\varphi, u, \widetilde{v}-\epsilon\} & \text { for }|\zeta| \leq 2 \text { and }\|z\| \leq \rho \\ \max \{\varphi, u\} & \text { elsewhere }\end{cases}
$$

This proves the lemma.

To prove Theorem 7.3, suppose first that $\Lambda_{K}^{0}$ is bounded on $D$ and let $\left\{u_{m}\right\}_{m=1}^{\infty}$ be a monotone increasing sequence from $\mathcal{L}_{K}$ converging to $\Lambda_{K}^{0}$ on $D$. For each $u_{m}$ let $u_{m, \epsilon_{m}}$ be the function given by Lemma 7.4 with $\epsilon_{m}=1 / m$. Then on $D$ we have

$$
u_{m}-\frac{1}{m} \leq \widetilde{v}_{m}-\frac{1}{m} \leq u_{m, \epsilon_{m}} \leq \Lambda_{K}^{0} .
$$

Thus $\Lambda_{K}^{0}$ is the limit of the monotone sequence of harmonic functions $\left\{\widetilde{v}_{m}\right\}_{m}$ and must be harmonic.

Suppose now that $\Lambda_{K}^{0}$ is unbounded on $D^{\prime}=\left\{\zeta \in D:|\zeta| \leq \frac{1}{4}\right\}$. Then there are sequences $\zeta_{m} \in D^{\prime}$ and $u_{m} \in \mathcal{L}_{K}$ with $u_{m}\left(\zeta_{m}\right) \geq m$. From (7.1) and the Harnak inequality we conclude that $\Lambda_{K}^{0} \equiv \infty$ on $D^{\prime}$. This completes the proof. 
THEOREM 7.5. The last assertion of Theorem 7.3 holds if the variety $Z$ has the property that for each point $p \in Z$ there is a neighborhood $\mathcal{O}$ of $p$ and an quasiplurisubharmonic function $\psi$ on $\mathbf{C}^{n}$ such that $Z \cap \mathcal{O}=\{x \in \mathcal{O}: \psi(x)=-\infty\}$.

Proof. Replace $\varphi$ in the argment above with the function $\psi+C$ for sufficiently large $C$.

Note. It is proved in [GZ, Thm. 5.2] that there always exists such a $\psi$ with

$$
Z \cap \mathcal{O} \subseteq\{x \in \mathcal{O}: \psi(x)=-\infty\} .
$$

If equality holds in (7.2) for some $\psi, Z$ is called completely pluripolar in $\Omega$. There are papers which describe how far a subvariety $Z \subset \Omega$ is from being completely pluripolar. See [Wie] for example.

8. The Theorem of Fabre. One might hope that projective hulls could be approached by studying polynomial hulls in affine open subsets of $\mathbf{P}^{n}$. This hope is essentially dashed by the following striking result of B. Fabre, whose proof uses the generalized Jacobians of singular curves introduced by M. Rosenlicht $[R]$.

TheOREM 8.1 (B. FABRe $\left[\mathrm{F}_{1}\right]$ ). Let $C \subset \mathbf{P}^{n}$ be an irreducible algebraic curve with non-empty singular set. Then there exist domains $\Omega \subset C$ with smooth boundary having the property that $\Omega$ meets every divisor in $\mathbf{P}^{n}$.

Note that the resulting Riemann surface with boundary $\Omega \subset \mathbf{P}^{n}$ has the property that it is not contained in any affine open subset of $\mathbf{P}^{n}$.

9. Examples. There are cases where we understand the projective hull completely.

Proposition 9.1. Let $Z \subset \mathbf{P}^{n}$ be an irreducible algebraic subvariety of $\mathbf{P}^{n}$, and suppose that $K \subset Z$ is a subset which is not pluripolar (equivalently, has positive capacity) in $Z$. Then $\widehat{K}=Z$.

Proof. Choose an affine chart $Z_{0} \equiv Z \cap \mathbf{C}^{n}$ and a compact subset $K_{0} \subset K \cap Z_{0}$ which is not pluripolar. It follows from [S, Prop. 2.1] that $\Lambda_{K_{0}}^{0}$ is locally bounded on $Z_{0}$. Therefore, by (6.8), $\Lambda_{K_{0}}$ (and so also $\Lambda_{K}$ ) is finite at all points of $Z_{0}$. Repeating the argument on slight perturbations of the chart shows that $\Lambda_{K}$ is finite on all of $Z$, i.e., $Z \subseteq \widehat{K}$. However, by Proposition 3.1 , we have $\widehat{K} \subseteq Z$.

Alternate Proof. Let $\Lambda_{K, Z}$ be the intrinsic extremal function for $K$ on the Kähler manifold $Z$ (with Kähler form induced from $\mathbf{P}^{n}$ ). Guedj and Zeriahi establish Theorem 4.3 for $\Lambda_{K, Z}$ on $Z$. However, by Proposition 17.4 we have $\left.\Lambda_{K}\right|_{Z}=\Lambda_{K, Z}$, and the result follows.

The projective hull of a subset $K \subset \mathbf{P}^{n}$ is not always an algebraic set.

Theorem 9.2. Let $V=\left\{(z, f(z)) \in \mathbf{C}^{2}: z \in \mathbf{C}\right\} \subset \mathbf{P}^{2}$ be the graph of an entire holomorphic function $f: \mathbf{C} \rightarrow \mathbf{C}$ which is not a polynomial. Then for any compact subset $K \subset V$ we have

$$
\widehat{K}=K \cup\{\text { the bounded components of } V-K\} \text {. }
$$

Proof. The bounded components of $V-K$ lie in the polynomial hull of $K$ and therefore in the projective hull $\widehat{K}$ by Corollary 3.4. By Theorem 7.1 the extremal 
function $\Lambda_{K}^{0}$ cannot be locally bounded on all of $V$ because $V$ is not algebraic. We shall prove that $\Lambda_{K}^{0} \equiv-\infty$ in $\mathbf{C}^{2}-V$. Then by Theorem 7.3 we must have $\Lambda_{K}^{0} \equiv-\infty$ on the unbounded component of $V-K$ and the assertion will follow.

It remains to prove that $\widehat{K} \subset V$. By applying a homothety to $\mathbf{C}^{2}$ we can assume that $\pi(K) \subset\{z \in \mathbf{C}:|z| \leq 1 / 2\}$ where $\pi: \mathbf{C}^{2} \rightarrow \mathbf{C}$ denotes projection onto the first coordinate. Since $f$ is entire, it has power series expansion $f(z)=\sum_{n=0}^{\infty} a_{n} z^{n}$ with

$$
\limsup _{n \rightarrow \infty}\left|a_{n}\right|^{\frac{1}{n}}=0 .
$$

Fix $\left(z_{0}, w_{0}\right) \notin V$ and consider now the family of polynomials $P_{d}(z, w)=w-\sum_{n=0}^{d} a_{n} z^{n}$ for $d \geq 1$. Since $w_{0} \neq f\left(z_{0}\right)$ we have

$$
\left|P_{d}\left(z_{0}, w_{0}\right)\right| \geq \frac{1}{2}\left|w_{0}-f\left(z_{0}\right)\right|>0 \quad \text { for all } d \text { sufficiently large. }
$$

However, since $K$ is contained in the graph of $f$ over $\left\{|z| \leq \frac{1}{2}\right\}$, we have

$$
\sup _{K}\left|P_{d}\right| \leq \sup _{|z| \leq \frac{1}{2}}\left\{\sum_{n=d+1}^{\infty}\left|a_{n}\right||z|^{n}\right\}<\sup _{n>d}\left|a_{n}\right|=\left\{\sup _{n>d}\left|a_{n}\right|^{\frac{1}{d}}\right\}^{d} \leq\left\{\sup _{n>d}\left|a_{n}\right|^{\frac{1}{n}}\right\}^{d}
$$

for large $d$, and so for any $C>0$ we have

$$
\lim _{d \rightarrow \infty} C^{d} \sup _{K}\left|P_{d}\right| \leq \lim _{d \rightarrow \infty} C^{d}\left\{\sup _{n>d}\left|a_{n}\right|^{\frac{1}{n}}\right\}^{d}=\lim _{d \rightarrow \infty}\left\{C \sup _{n>d}\left|a_{n}\right|^{\frac{1}{n}}\right\}^{d} \leq C \limsup _{n \rightarrow \infty}\left|a_{n}\right|^{\frac{1}{n}} .
$$

By (9.1) the rightmost term is zero. Hence there exists no constant $C>0$ such that $\left|P_{d}\left(z_{0}, w_{0}\right)\right| \leq C^{d} \sup _{K}\left|P_{d}\right|$ for all $d$, and so by Proposition $6.1\left(z_{0}, w_{0}\right) \notin \widehat{K}$. ing.

Further interesting examples come from classical gap series. We recall the follow-

Theorem 9.3 (See Hille [Hi]). Consider the holomorphic function

$$
f(z)=\sum_{k=0}^{\infty} c_{n_{k}} z^{n_{k}} \quad \text { with } \quad \limsup _{k \rightarrow \infty}\left\{\left|c_{n_{k}}\right|^{\frac{1}{n_{k}}}\right\}=1 .
$$

Assume that there exists $\lambda>1$ with $\lambda n_{k}<n_{k+1}$ for all $k>k_{0}$. Then $\Delta_{1}(0)=\{|z|<$ $1\}$ is the domain of analyticity of $f$

THEOREM 9.4. Let $f$ be as in Theorem 9.3 and assume the series (9.3) converges for all $|z|=1$. Fix any $r, 0<r<1$, and let

$$
V_{r} \equiv\left\{( z , f ( z ) \in \mathbf { C } ^ { 2 } : | z | < r \} \quad \text { and } \quad d V _ { r } \equiv \left\{\left(z, f(z) \in \mathbf{C}^{2}:|z|=r\right\} .\right.\right.
$$

If

$$
\limsup _{k \rightarrow \infty} \frac{n_{k+1}}{n_{k}}=\infty
$$

then for all $r$,

$$
\widehat{d V_{r}}=V_{r}
$$


Proof. As in the proof of Theorem 9.2 it will suffice to prove that $\widehat{d V_{r}} \subseteq V_{1}$. To see this, fix $z_{0} \in \bar{\Delta}_{1}$ and choose $w_{0} \neq f\left(z_{0}\right)$. Set $P_{n_{k}}(z, w)=w-\sum_{j=1}^{k} c_{n_{j}} z^{n_{j}}$ and write $P_{n_{k}}(z, w)=w_{0}-f\left(z_{0}\right)+\sum_{j=k+1}^{\infty} c_{n_{j}} z_{0}^{n_{j}}$. Then for all $k$ sufficiently large we have

$$
\left|P_{n_{k}}(z, w)\right| \geq\left|w_{0}-f\left(z_{0}\right)\right|-\left|\sum_{j=k+1}^{\infty} c_{n_{j}} z_{0}^{n_{j}}\right| \geq>0 .
$$

On the other hand, using (9.3) above we find that

$$
\begin{aligned}
\sup _{d V_{r}} & =\sup _{|z|=r}\left|f(z)-\sum_{j=1}^{k} c_{n_{j}} z^{n_{j}}\right| \\
& =\sup _{|z|=r}\left|\sum_{j=k+1}^{\infty} c_{n_{j}} z^{n_{j}}\right| \leq \sum_{j=k+1}^{\infty}\left(\left|c_{n_{j}}\right|^{\frac{1}{n_{j}}} r\right)^{n_{j}} \\
& \leq \sum_{j=k+1}^{\infty}\left(\sup _{\ell \geq j}\left|c_{n_{\ell}}\right|^{\frac{1}{n_{\ell}}} r\right)^{n_{j}} \leq \sum_{j=k+1}^{\infty}(\sqrt{r})^{n_{j}}<L r^{\frac{n_{k+1}}{2}}
\end{aligned}
$$

for $L=(1-\sqrt{r})^{-1}$ and for all $k$ sufficiently large.

Now if $\left(z_{0}, w_{0}\right) \in \widehat{d V_{r}}$, then by Proposition 6.1 and the paragraph above, there exists $C>1$ with

$$
K \leq C^{n_{k}} \operatorname{Lr} \frac{n_{k+1}}{2} .
$$

Hence, there exists $K_{0}>0, a>0$ and $b>0$ with

$$
K_{0} \leq \exp \left(a n_{k}-b n_{k+1}\right) \quad \text { for all } k \text { sufficiently large. }
$$

Let $\kappa_{0}=\log \left(K_{0}\right)$. Then

$$
\kappa_{0} \leq a n_{k}-b n_{k+1} \quad \text { for all } k \text { sufficiently large, }
$$

or equivalently

$$
\frac{n_{k+1}}{n_{k}}+\frac{\kappa_{0}}{b n_{k}} \leq \frac{a}{b}
$$

which contradicts assumption (9.4). We conclude that $\left(z_{0}, w_{0}\right) \notin \widehat{d V_{r}}$.

Suppose now that $\left|z_{0}\right|=R>1$ and $w_{0}$ is arbitrary. Set $P_{n_{k}}(z, w)=w-$ $\sum_{j=1}^{k} c_{n_{j}} z^{n_{j}}$ as before and note that by (9.3)

$$
\begin{aligned}
\left|P_{n_{k}}\left(z_{0}, w_{0}\right)\right| & \geq\left|c_{n_{k}}\right| R^{n_{k}}-\left|w_{0}\right|-\sum_{j=1}^{k-1}\left|c_{n_{j}} z_{0}^{n_{j}}\right| \\
& \geq\left(\left|c_{n_{k}}\right|^{\frac{1}{n_{k}}} R\right)^{n_{k}}-\left|w_{0}\right|-K \sum_{j=1}^{k-1}(\rho R)^{n_{j}}
\end{aligned}
$$

for all $k$ is sufficiently large, where $\rho$ satisfies $1<\rho<R$. Choose $\alpha<1$ with $\rho<\alpha^{2} R$. 
Then for $k$ sufficiently large

$$
\begin{aligned}
\left(\left|c_{n_{k}}\right|^{\frac{1}{n_{k}}} R\right)^{n_{k}}-\left|w_{0}\right|-K \sum_{j=1}^{k-1}(\rho R)^{n_{j}} & \geq(\alpha R)^{n_{k}}-K_{1} \sum_{m=0}^{n_{k-1}}(\rho R)^{m} \\
& =(\alpha R)^{n_{k}}-K_{1} \frac{(\rho R)^{n_{k-1}+1}-1}{\rho R-1} \\
& \geq \frac{(\alpha R)^{n_{k}}(\rho R-1)-K_{1}(\rho R)^{n_{k-1}+1}}{\rho R-1} .
\end{aligned}
$$

The numerator of the last term in (9.5) is

$$
\begin{aligned}
& {\left[\left(\frac{\alpha}{\rho}\right)^{n_{k-1}+1}(\alpha R)^{n_{k}-n_{k-1}-1}(\rho R-1)-K_{1}\right](\rho R)^{n_{k-1}+1}} \\
& \geq\left(\frac{\alpha}{\rho}\right)^{n_{k-1}}(\alpha R)^{n_{k}-n_{k-1}-1}(\rho R-1)-K_{1} \\
& \geq\left(\frac{\alpha^{2} R}{\rho}\right)^{n_{k-1}}(\alpha R)^{n_{k}-2 n_{k-1}-1}(\rho R-1)-K_{1} \\
& \geq(\alpha R)^{n_{k}-2 n_{k-1}-1}(\rho R-1)-K_{1} \longrightarrow \infty
\end{aligned}
$$

since $\alpha R>\alpha^{2} R / \rho>1$ and $n_{k}-2 n_{k-1} \rightarrow \infty$. In particular $\left|P_{n_{k}}\left(z_{0}, w_{0}\right)\right| \geq K_{2}>0$ for all $k$ sufficiently large, and our previous estimate for $\sup _{d V_{r}}\left|p_{n_{k}}\right|$ rules this case out as well.

Remark 9.4. (A Projectively Convex Curve). We claim that for the curve

$$
\Gamma=\left\{(z, \exp (z+\bar{z}):|z|=1\} \subset \mathbf{C}^{2} \subset \mathbf{P}^{1} \times \mathbf{P}^{1} \subset \mathbf{P}^{3}, \text { one has } \widehat{\Gamma}=\Gamma .\right.
$$

An outline of the proof is as follows. Arguing as in Theorem 9.2 one sees that $\widehat{\Gamma}$ is contained in

$$
Z=\left\{(z, w): w=\exp \left(z+\frac{1}{z}\right), \quad 0<|z|<\infty\right\} \cup\left(0 \times \mathbf{P}^{1}\right) \cup\left(\infty \times \mathbf{P}^{1}\right) .
$$

By Theorem 7.3 the extremal function $\Lambda_{\Gamma}^{0}$ (and therefore also $\Lambda_{\Gamma}$ ) is either $\equiv \infty$ or is a locally bounded function on each of the two components of $Z$ over $\{0<|z|<1\}$ and $\{1<|z|<\infty\}$. However, the automorphism $(z, w) \mapsto(1 / z, w)$ shows that either $\Lambda_{\Gamma}^{0} \equiv \infty$ on both components or it is bounded on both. However, by Sadullaev' Theorem 7.1 it cannot be bounded on both, and therefore

$$
\widehat{\Gamma} \subseteq \Gamma \cup\left(0 \times \mathbf{P}^{1}\right) \cup\left(\infty \times \mathbf{P}^{1}\right) .
$$

Suppose now that there exists a point $x \in \widehat{\Gamma} \cap\left(0 \times \mathbf{P}^{1}\right)$. By Theorem 11.1 there exists a probability measure $\mu$ on $\Gamma$ and a positive current $T$ of bidimension $(1,1)$ with support in $\widehat{\Gamma}$, such that $d d^{c} T=\mu-\delta_{x}$. This is impossible since, if $\pi: \mathbf{P}^{1} \times \mathbf{P}^{1} \rightarrow \mathbf{P}^{1}$ denotes projection on the first factor, we would have $d d^{c} \pi_{*} T=\pi_{*} \mu-\delta_{0}$ with supp $\left(\pi_{*} T\right)$ in the unit circle $S^{1}$ and $\pi_{*} \mu$ a probablilty measure on $S^{1}$. By symmetry we conclude that $\widehat{\Gamma}=\Gamma$.

10. Compactness and Stability. While we have many representations of the projective hull, we do not have an easy answer to the following natural question: Given 
a compact subset $K \subset \mathbf{P}^{n}$, is $\widehat{K}$ also compact? Proposition 9.1 shows that if $K$ is contained in an affine chart $\mathbf{C}^{n}$, its projective hull may not be contained in that chart. For example consider the tiny curve

$$
K=\left\{\left(t,(1+t)^{\frac{1}{m}}\right) \in \mathbf{C}^{2}:|t|=\epsilon\right\}
$$

for some choice of $m$ th root and $\epsilon$ very small. Then $\widehat{K} \cap \mathbf{C}^{2}=\left\{(x, y): y^{m}=1+x\right\}$ is an algebraic curve which is $m$-sheeted over the entire $x$-axis.

Definition 10.1. A subset $K \subset \mathbf{P}^{n}$ is called stable if the best constant function $C: \widehat{K} \rightarrow \mathbf{R}^{+}$is bounded above, or equivalently, the radius function $\rho_{K}: \widehat{K} \rightarrow \mathbf{R}^{+}$ defined in (5.4) is bounded below by a positive constant (cf. Proposition 5.2).

Note that $\widehat{S(K)}_{\text {poly }}$ has an outer boundary consisting of all points $Z \in \widehat{S(K)}_{\text {poly }}-$ $\{0\}$ with $\|Z\|=\rho(\pi Z)$. Stability is equivalent to the fact that 0 is not in the closure of this outer boundary.

Proposition 10.2. If $K \subset \mathbf{P}^{n}$ is stable, then $\widehat{K}$ is compact.

Proof. If $C_{K}(x) \leq C<\infty$ for all $x \in \widehat{K}$, then for every section $\mathcal{P}$ of $\mathcal{O}(d)$

$$
\|\mathcal{P}(x)\| \leq C^{d} \sup _{K}\|\mathcal{P}\| \quad \text { for all } x \in \widehat{K}
$$

and hence for all $x$ in the closure of $\widehat{K}$, proving that $\widehat{K}$ is closed.

We next examine some elementary local properties of projective hulls.

Fix a closed set $K \subset \mathbf{P}^{n}$.

Proposition 10.3. Suppose $B \subset \mathbf{P}^{n}$ is a closed subset with $C_{K}$ bounded above on $\widehat{K} \cap B$. Then $\widehat{K} \cap B$ is compact and

$$
(\widehat{K} \cap B)^{\wedge} \cap B=\widehat{K} \cap B .
$$

In particular if $\widehat{K}$ is stable, then

$$
\widehat{\widehat{K}}=\widehat{K}
$$

Proof. The compactness is proved as in 10.2. For the second assertion it suffices to show that $(\widehat{K} \cap B)^{\wedge} \subseteq \widehat{K}$. By assumption there exists a constant $C<\infty$ with

$$
\sup _{\widehat{K} \cap B}\|\mathcal{P}\| \leq C^{d} \sup _{K}\|\mathcal{P}\|
$$

for all $\mathcal{P} \in H^{0}\left(\mathbf{P}^{n}, \mathcal{O}(d)\right)$ and all $d \geq 0$. If $x \in(\widehat{K} \cap B)^{\wedge}$, there exists $C_{x}$ such that

$$
\|\mathcal{P}(x)\| \leq C_{x}^{d} \sup _{\widehat{K} \cap B}\|\mathcal{P}\| \leq\left(C_{x} C\right)^{d} \sup _{K}\|\mathcal{P}\|
$$

for all $\mathcal{P}, d$ as above. Hence, $x \in \widehat{K}$.

We now switch to affine coordinates.

Proposition 10.4. Suppose $B$ is a compact polynomially convex subset of $\mathbf{C}^{n} \subset$ $\mathbf{P}^{n}$. If $C_{K}$ is bounded on $\widehat{K} \cap B$, then $\widehat{K} \cap B$ is polynomially convex. 
Proof. Set

$$
C_{B} \equiv \sup _{z \in \widehat{K} \cap B} C(z)\left(1+|z|^{2}\right)^{\frac{1}{2}}<\infty .
$$

Then it follows directly from (6.3) that for any polynomial $p \in \mathbf{C}\left[z_{1}, \ldots, z_{n}\right]$ of degree $d$

$$
\sup _{\widehat{K} \cap B}|p(z)| \leq C_{B}^{d} \sup _{\widehat{K}}\|\mathcal{P}\|
$$

where $\mathcal{P}$ is the associated section of $\mathcal{O}(d)$. Now if $x \in[\widehat{\widehat{K} \cap B}]_{\text {poly }}$, then

$$
\frac{|p(z)|}{\left(1+|z|^{2}\right)^{\frac{d}{2}}} \leq|p(z)| \leq \sup _{\widehat{K} \cap B}|p| \leq C_{B}^{d} \sup _{\widehat{K}}\|\mathcal{P}\|
$$

for all polynomials $p$ as above, and so $x \in \widehat{K}$. Furthermore,

$$
|p(z)| \leq \sup _{\widehat{K} \cap B}|p| \leq \sup _{B}|p|
$$

and so $x \in \widehat{B}_{\text {poly }}=B$. Hence $x \in \widehat{K} \cap B$.

11. Projective Hulls and Jensen Measures - The Main Result. In this section we shall prove our central analytic result concerning projective hulls. A general version of the theorem will be established in $\S 18$, but here we shall work on $\mathbf{P}^{n}$. Let

$$
P \equiv \mathcal{P S} \mathcal{H}_{\omega}=\left\{\varphi \in C\left(\mathbf{P}^{n}\right): d d^{c} \varphi+\omega \geq 0\right\}
$$

be the space of continuous quasi-plurisubharmonic functions where $\omega$ is the standard Kähler form. Then the projective hull of a compact subset $K \subset \mathbf{P}^{n}$ is the set $\widehat{K}$ of points $x$ for which there exists a constant $\lambda=\lambda_{x}$ such that

$$
\varphi(x) \leq \lambda+\sup _{K} \varphi \quad \text { for all } \varphi \in P .
$$

Denote by $\widehat{K}(\Lambda)$ the subset of $\widehat{K}$ for which there exists a $\lambda \leq \Lambda$ satisfying condition (11.1). Let $\mathcal{P}_{1,1}(\Lambda)$ denote the convex cone of positive currents of bidimension $(1,1)$ and mass $\leq \Lambda$ on $\mathbf{P}^{n}$, and $\mathcal{M}_{K}$ the set of probability measures on $\mathbf{P}^{n}$ with support in $K$.

THEOREM 11.1. For a compact subset $K \subset \mathbf{P}^{n}$ the following are equivalent:

(A) $\quad x \in \widehat{K}(\Lambda)$

(B) There exist $T \in \mathcal{P}_{1,1}(\Lambda)$ and $\mu \in \mathcal{M}_{K}$ such that:

$$
\begin{aligned}
& \text { (i) } d d^{c} T=\mu-\delta_{x} \\
& \text { (ii) } \operatorname{supp}(T) \subset \widehat{K}^{-}
\end{aligned}
$$

Proof. What follows is a succinct proof of the result. In $\S 18$ a more rounded and geometric proof is given of a more general result. The interested reader may want to go directly there.

We first show that $(\mathrm{B}) \Rightarrow(\mathrm{A})$. Suppose $T \in \mathcal{P}_{1,1}(\Lambda)$ satisfies (i) and (ii). Let $\varphi \in \mathcal{P S H}_{\omega}$, so that

$$
d d^{c} \varphi+\omega=\eta \geq 0 .
$$


Then

$$
\begin{aligned}
\int_{K} \varphi d \mu-\varphi(x) & =\left(d d^{c} T\right)(\varphi)=T\left(d d^{c} \varphi\right)=T(\eta)-T(\omega) \\
& \geq-T(\omega)=-M(T) \geq-\Lambda .
\end{aligned}
$$

Hence, $\varphi(x) \leq \sup _{K} \varphi+\Lambda$, and we conclude that $x \in \widehat{K}(\Lambda)$.

To show that $(\mathrm{A}) \Rightarrow(\mathrm{B})$ we shall need the following.

Lemma 11.2. Fix a compact subset $B \subset \mathbf{P}^{n}-\widehat{K}^{-}$and real numbers $M, N$ with $N-M>0$. Then for all $\Lambda>0$ there exists a function $\varphi \in P$ such that $\left.\varphi\right|_{\widehat{K}(\Lambda)} \leq M$ and $\left.\varphi\right|_{B} \geq N$.

Proof. Condition (11.1) and compactness enables us to find a finite set $\varphi_{1}, \ldots, \varphi_{m} \in$ $P$ such that $\sup _{K} \varphi_{i} \leq M-\Lambda$ for all $i$ and $\varphi \equiv \max \left\{\varphi_{1}, \ldots, \varphi_{m}\right\} \geq N$ on $B$.

We now fix $\epsilon>0$ and consider the closed convex cone

$$
\mathcal{P}_{1,1}(\Lambda, \epsilon) \equiv\left\{T \in \mathcal{P}_{1,1}(\Lambda): \operatorname{supp}(T) \subset \widehat{K}_{2 \epsilon}\right\}
$$

where

$$
\widehat{K}_{t}=\left\{x \in \mathbf{P}^{n}: \operatorname{dist}(x, \widehat{K}) \leq t\right\} .
$$

It will suffice to show that there exists a current $T \in \mathcal{P}_{1,1}(\Lambda, \epsilon)$ satisfying conditions (i) and (ii) above. If this is not the case, i.e., if

$$
\left(\mathcal{M}_{K}-\delta_{x}\right) \cap d d^{c} \mathcal{P}_{1,1}(\Lambda, \epsilon)=\emptyset
$$

then by the Hahn-Banach Theorem there exists $\varphi \in C^{\infty}\left(\mathbf{P}^{n}\right)$ and $\gamma \in \mathbf{R}$ such that

$$
\sup _{\mu \in \mathcal{M}_{K}}\left(\mu-\delta_{x}\right)(\varphi)<\gamma<\left(d d^{c} T\right)(\varphi)
$$

for all $T \in \mathcal{P}_{1,1}(\Lambda, \epsilon)$. Note that since $0 \in \mathcal{P}_{1,1}(\Lambda, \epsilon)$ we have $\gamma<0$. Setting $\psi=\frac{\Lambda}{|\gamma|} \varphi$, we find that

$$
\sup _{\mu \in \mathcal{M}_{K}}\left(\mu-\delta_{x}\right)(\psi)<-\Lambda<\left(d d^{c} T\right)(\psi)=T\left(d d^{c} \psi\right)
$$

for all $T \in \mathcal{P}_{1,1}(\Lambda, \epsilon)$. Applying the right hand inequality to currents of the form $T=\delta_{y} \xi$ where $y \in \widehat{K}_{2 \epsilon}$ and $\xi$ is a positive simple $(1,1)$ vector of length $\Lambda$ at $y$, we conclude that

$$
d d^{c} \psi+\omega \geq 0 \quad \text { on } \widehat{K}_{2 \epsilon} .
$$

Now let $\varphi_{M, N} \in P$ be the function given by Lemma 11.2 with $M$ and $N$ chosen so that

$$
M<\inf _{\widehat{K}(\Lambda)} \psi \quad \text { and } \quad N>\sup _{\mathbf{P}^{n}-\widehat{K}_{\epsilon}^{0}} \psi
$$

Then the function

$$
\Psi \equiv \max \left\{\psi, \varphi_{M, N}\right\}
$$

has the property that

$$
\Psi=\psi \quad \text { on } \widehat{K}(\Lambda) \quad \text { and } \quad \Psi=\varphi_{M, N} \quad \text { on } \mathbf{P}^{n}-\widehat{K}_{\epsilon}^{0} .
$$


Consequently, $d d^{c} \Psi+\omega \geq 0$ on all of $\mathbf{P}^{n}$, that is, $\Psi \in P$. Furthermore, by (11.2) and (11.3) we have that

$$
\sup _{\mu \in \mathcal{M}_{K}} \int_{K} \Psi d \mu-\Psi(x)<-\Lambda
$$

for all probablility measures $\mu$ on $K$. Choosing $\mu=\delta_{y}$ for $y \in K$ shows that

$$
\sup _{K} \Psi-\Psi(x)<-\Lambda
$$

which means that $x \notin \widehat{K}(\Lambda)$.

Corollary 11.3. For any $\nu \in \mathcal{M}_{\widehat{K}(\Lambda)}$ there exists $T \in \mathcal{P}_{1,1}(\Lambda)$ and $\mu \in \mathcal{M}_{K}$ with

$$
\begin{aligned}
& \text { (i) } \quad d d^{c} T=\mu-\nu \\
& \text { (ii) } \operatorname{supp}(T) \subset \widehat{K}^{-} .
\end{aligned}
$$

Proof. The probability measures on $\widehat{K}(\Lambda)$ are the closed convex hull of the $\delta$ measures.

Corollary 11.4. For any $x \in \widehat{K}^{-}$there are probability measures $\nu \in \mathcal{M}_{\widehat{K}^{-}}$, $\mu \in \mathcal{M}_{K}$ and a current $T \in \mathcal{P}_{1,1}$ with

$$
\begin{aligned}
& \text { (i) } d d^{c} T=\mu-\nu \\
& \text { (ii) } x \in \operatorname{supp}(T) \subset \widehat{K}^{-} .
\end{aligned}
$$

Proof. Let $\left\{x_{k}\right\}_{k=1}^{\infty} \subset \widehat{K}$ be a sequence converging to $x$. Choose currents $T_{k} \in$ $\mathcal{P}_{1,1}$ as in Theorem 11.1 with $d d^{c} T_{k}=\mu_{k}-\delta_{x_{k}}$ and $\operatorname{supp}\left(T_{k}\right) \subset \widehat{K}^{-}$. Then the positive current

$$
\widetilde{T} \equiv \sum_{k=1}^{\infty} \frac{1}{2^{k} M\left(T_{k}\right)} T_{k}
$$

has $x \in \operatorname{supp}(T)$ and satisfies $d d^{c} \widetilde{T}=\widetilde{\mu}-\widetilde{\nu}$ for positive measures $\widetilde{\mu}$ on $K$ and $\widetilde{\nu}$ on $\widehat{K}^{-} . T \equiv \frac{1}{\mu(K)} \widetilde{T}$ is the desired current.

REMARK 11.5. Let $\Lambda_{K}(x)$ be the extremal function introduced in (1.2) and discussed in $\S 4$. Note that by definition:

$$
x \in \widehat{K}(\Lambda) \Leftrightarrow \Lambda_{K}(x) \leq \Lambda .
$$

For a fixed point $x \in \widehat{K}$ let $\mathcal{F}_{x}$ denote the set of positive currents $T$ of bidimension $(1,1)$ satisfying $d d^{c} T=\mu-\delta_{x}$ for some $\mu \in \mathcal{M}_{K}$ and set $\mathcal{N}_{K}=\left\{T \in \mathcal{M}_{K}: \operatorname{supp}(T) \subseteq\right.$ $\left.\widehat{K}^{-}\right\}$. Let $T_{x}$ be the current guaranteed by Theorem 11.1. Then from the discussion above we have that

$$
\Lambda_{K}(x)=M\left(T_{x}\right)=\inf _{\mathcal{M}_{K}} M(T)=\inf _{\mathcal{N}_{K}} M(T) .
$$

In other words $T_{x}$ is the positive current of least mass satisfying the equation $d d^{c} T=$ $\mu-\delta_{x}$, and that least mass is exactly $\Lambda_{K}(x)$.

The middle equality in (11.4) follows from the fact that Theorem 11.1 also holds without the requirement supp $(T) \subseteq \widehat{K}^{-}$as one can easily check (cf. Theorem 18.2 below). 
REMARK 11.6. Suppose that $K=\gamma$ is a closed curve and $\widehat{K}=V$ is a 1dimensional complex submanifold with boundary $\gamma$ as in the examples below. Then

$$
T_{x}=G_{x}[V]
$$

where $G_{x}$ is the Green's function on $V$ with singularity at $x$.

12. Structure Theorems. Theorem 11.1 has a number of basic consequences. We recall that a subset $W$ of a complex manifold $Z$ is called 1-concave if for every open set $\mathcal{O} \subset \subset Z$ and every holomorphic map $f$ from a neighborhood of $\overline{\mathcal{O}}$ to $\mathbf{C}$ one has $f(W \cap \mathcal{O}) \subset \mathbf{C}-\Omega$ where $\Omega$ is the unbounded component of $\mathbf{C}-f(W \cap \partial \mathcal{O})$.

Fix a compact subset $K \subset \mathbf{P}^{n}$

Theorem 12.1. The set $\widehat{K}^{-}-K$ is 1-concave in $\mathbf{P}^{n}-K$.

Note. It follows that $\left(\widehat{K}^{-}-K\right) \cap O$ is 1 -concave in $O-K$ for any open subset $O \subset \mathbf{P}^{n}$.

Proof. Corollary 11.4 implies that for any $x \in \widehat{K}^{-}-K$ there exists a positive $(1,1)$-current $T$ with

$$
\begin{aligned}
& \text { (i) } \quad d d^{c} T=-\nu \leq 0 \quad \text { in } \mathbf{P}^{n}-K \quad \text { and } \\
& \text { (ii) } \quad x \in \operatorname{supp}(T) \subseteq \widehat{K}^{-} .
\end{aligned}
$$

By Proposition 2.2 of $[\mathrm{DL}]$ we conclude that $\operatorname{supp}(T)$ is 1 -concave in $\mathbf{P}^{n}-K$. The argument given for Proposition 2.3 of [DL] now gives the following:

Lemma 12.2. . Let $\mathcal{S}$ denote the family of (closed) 1-concave subsets of $\mathbf{P}^{n}-K$ which have support in $\widehat{K}^{-}$. Then the union of all elements in $\mathcal{S}$ is again an element of $\mathcal{S}$.

By (12.1) this maximal 1-concave subset equals $\widehat{K}^{-}$.

Corollary 12.3. For any compact subset $K \subset \mathbf{P}^{n}$ the closed projective hull $\widehat{K}^{-}-K$ has locally positive Hausdorff 2-measure.

Proof. Any 1-concave subset of a complex manifold has positive Hausdorff 2measure in any neighborhood of any point. (Otherwise the complement of the image under any holomorphic map to $\mathbf{C}$ would be connected.)

TheOREM 12.4. Let $K \subset \mathbf{P}^{n}$ be any compact subset and assume that the Hausdorff 2-measure of $\widehat{K}^{-}$is locally finite. Then $\widehat{K}^{-}-K$ is a 1-dimensional analytic subvariety of $\mathbf{P}^{n}-K$.

Moreover, suppose $D \subset \mathbf{C}^{n}-K \subset \mathbf{P}^{n}$ is a strictly convex domain with smooth boundary. If the Hausdorff 1-measure of $\widehat{K}^{-} \cap \partial D$ is finite, then $\widehat{K}^{-} \cap D$ is a 1dimensional analytic subvariety of $D$.

Proof. This follows from Theorem 12.1 and [DL, Thm. 3.3 and Cor. 3.8]. It also follows from Theorem 5.7 and [Sib, Thm. 17].

THEOREM 12.5. Let $\Gamma \subset \mathbf{P}^{2}$ be a finite union of smooth closed curves which are pluripolar. Then the local Hausdorff dimension of $\widehat{\Gamma}$ is everywhere 2. The conclusion also holds for any $\Gamma \subset \mathbf{P}^{n}$ which is real analytic. 
Proof. Since $\Gamma$ is pluripolar, $\widehat{\Gamma}$ is pluripolar by Corollary 4.4. Suppose $\mathcal{H}^{2+\alpha}(\widehat{\Gamma})>$ 0 for some $\alpha>0$ where $\mathcal{H}^{\beta}$ denotes Hausdorff measure in dimension $\beta$. Applying the coarea formula $[\mathrm{F}, 3.2]$ to a linear projection $\pi: \mathbf{P}^{2}-\mathbf{P}^{0} \rightarrow \mathbf{P}^{1}$ shows that the set of $y \in \mathbf{P}^{1}$ with $\mathcal{H}^{\alpha}\left(\widehat{\Gamma} \cap \pi^{-1}(y)\right)>0$ has positive $\mathcal{H}^{2}$-measure.

Now the condition $\mathcal{H}^{\alpha}\left(\widehat{\Gamma} \cap \pi^{-1}(y)\right)>0$ implies that $\pi^{-1}(y) \subset \widehat{\Gamma}$. To see this consider the sets $\widehat{\Gamma}_{y, t} \equiv\left\{x \in \widehat{\Gamma} \cap \pi^{-1}(y): C_{\Gamma} \leq t\right\}$, and note that $\mathcal{H}^{\alpha}\left(\widehat{\Gamma}_{y, t}\right)>0$ for $t$ sufficiently large. For such $t$, ProjHull $\left(\widehat{\Gamma}_{y, t}\right)=\pi^{-1}(y)$ since sets of capacity zero in $\mathbf{P}^{1}$ have measure zero. However, ProjHull $\left(\widehat{\Gamma}_{y, t}\right) \subset \widehat{\Gamma}$, and so $\pi^{-1}(E) \subset \widehat{\Gamma}$ for some set $E$ of positive measure in $\mathbf{P}^{1}$. Hence, $\mathcal{H}^{4}(\widehat{\Gamma})>0$ and therefore $\widehat{\Gamma}=\mathbf{P}^{2}$ contradicting the pluripolarity of $\widehat{\Gamma}$.

To prove the second statement note that any real analytic curve is pluripolar and that under projections $\pi: \mathbf{P}^{n}-\mathbf{P}^{n-3} \rightarrow \mathbf{P}^{2}$ one has $\pi(\widehat{\Gamma}) \subseteq \widehat{\pi(\Gamma)}$

THEOREM 12.6. Suppose that $K \subset \subset \mathbf{C}^{n}$ and let $\widehat{K}_{0}$ be a connected component of $\widehat{K}^{-}-K$ which is bounded in $\mathbf{C}^{n}$. Then $\widehat{K}_{0}$ is contained in the polynomial hull of $K$.

Proof. Since $\widehat{K}^{-}-K$ is a 1 -concave subset of $\mathbf{P}^{n}-K$, so is any connected component $\widehat{K}_{0}$. We now use the fact ([DL, Prop.2.5]) that the polynomial hull of $K$ in $\mathbf{C}^{n}$ is the union of all bounded, 1-concave subsets of $\mathbf{C}^{n}-K$.

COROLlary 12.7. If $\widehat{K} \subset \Omega=\mathbf{P}^{n}-D$ for some algebraic hypersurface $D$, then $\widehat{K}=\widehat{K}_{\Omega}$. In particular if $\gamma \subset \Omega$ is a $C^{1}$-curve and if $\widehat{\gamma} \subset \subset \Omega$, then $\widehat{\gamma}-\gamma$ is a 1-dimensional analytic subvariety of $\Omega-\gamma$.

Proof. By Proposition 3.2 taking projective hulls commutes with Veronese embeddings. However, by embedding $\mathbf{P}^{n} \subset \mathbf{P}^{N}$ by the $d$ th Veronese map, where $d=$ $\operatorname{deg}(D)$, we reduce to the situation of Theorem 12.6.

The next result is a strong form of the Local Maximum Principle for projective hulls.

TheOREM 12.8. Fix $U^{\text {open }} \subset \subset \Omega^{\text {affine open }} \subset \mathbf{P}^{n}$. Then

$$
\widehat{K}^{-} \cap U \subseteq \text { PolyHull }\left\{\left(\widehat{K}^{-} \cap \partial U\right) \cup(K \cap U)\right\}
$$

with equality if $C_{K}$ is bounded on $\widehat{K} \cap \bar{U}$.

Proof. Set

$$
\begin{gathered}
\Sigma \equiv \widehat{K}^{-}-K \\
O \equiv \mathbf{P}^{n}-\left(\widehat{K}^{-} \cap \partial U\right)
\end{gathered}
$$

By the Note following Theorem 12.1, $\Sigma \cap O$ is 1-concave in $O-K$.

Claim: $\Sigma \cap U$ is a union of connected components of $\Sigma \cap O$.

Proof. Set $\Sigma_{O} \equiv \Sigma \cap O$. Note that

$$
\mathbf{P}^{n}=U \amalg \partial U \amalg(\sim \bar{U})
$$


gives

$$
\begin{aligned}
\Sigma_{O} & =\left(\Sigma_{O} \cap U\right) \amalg\left(\Sigma_{O} \cap \partial U\right) \amalg\left(\Sigma_{O} \cap \sim \bar{U}\right) \\
& =\left(\Sigma_{O} \cap U\right) \amalg\left(\Sigma_{O} \cap \sim \bar{U}\right)
\end{aligned}
$$

since

$$
\begin{aligned}
\Sigma_{O} \cap \partial U & =(\Sigma \cap O) \cap \partial U \\
& =\left(\widehat{K}^{-}-K\right) \cap\left(\mathbf{P}^{n}-\left(\widehat{K}^{-} \cap \partial U\right)\right) \cap \partial U \\
& =\left\{\widehat{K}^{-}-K-\left(\widehat{K}^{-} \cap \partial U\right)\right\} \cap \partial U \\
& =\emptyset .
\end{aligned}
$$

Therefore (12.2) gives a disconnection of $\Sigma_{O}$. This proves the claim.

Now by [DL] we know that for any compact subset $C \subset \Omega$ we have that the polynomial hull of $C$ equals the 1-Hull of $C$ which is by definition the union of all bounded 1-concave subsets of $\Omega-C$.

For the last statement recall from Proposition 10.3 that if $C_{K}$ is bounded on $\widehat{K} \cap \bar{U}$, then $(\widehat{K} \cap \bar{U})^{\wedge} \cap \bar{U}=\widehat{K} \cap \bar{U}$. Now use the fact that for bounded subsets of $\Omega$, the polynomial hull is contained in the projective hull.

This enables us to give the following generalization of Wermer's Theorem.

THEOREM 12.9. Let $\gamma \subset \mathbf{P}^{n}$ be a finite union of real analytic curves. Then $\widehat{\gamma}$ is a subset of Hausdorff dimension 2 whose closure is 1-concave. Furthermore, if the Hausdorff 2-measure of $\widehat{\gamma}^{-}$is finite in a neighborhood of some complex hypersurface, then $\widehat{\gamma}-\gamma=\widehat{\gamma}^{-}-\gamma$ is a 1-dimensional complex analytic subvariety of $\mathbf{P}^{n}-\gamma$.

The same conlcusion holds for any smooth pluripolar curve $\gamma$ in $\mathbf{P}^{2}$.

Proof. Theorems 12.1 and 12.5 give the first statement. Suppose now that $\mathcal{H}^{2}\left(\widehat{\gamma}^{-} \cap\right.$ $\mathcal{O})<\infty$ where $\mathcal{O}$ is a neighborhood of some divisor $D$. We may assume that $D \cap \gamma=\emptyset$ and therefore that $\mathcal{O} \cap \gamma=\emptyset$. By Theorem 12.4 we know that $\widehat{\gamma}^{-} \cap \mathcal{O}$ is a 1 dimensional complex analytic subvariety of $\mathcal{O}$. We now choose a bounded subdomain $U \subset \subset \Omega=\mathbf{P}^{n}-D$ with real analytic boundary $\partial U \subset \subset \mathcal{O}$. Then $\Gamma \equiv\left(\widehat{\gamma}^{-} \cap \partial U\right) \cup \gamma$ is a real analytic curve (which we may assume to be regular by appropriate choice of $U$ ), and by Wermer's Theorem $\widehat{\Gamma}$ is a bounded 1-dimensional complex subvariety of $\Omega-\Gamma$ with regularity at the boundary as in $\left[\mathrm{HL}_{1}\right]$. In particular it is regularly and analytically immersed up to the boundary in $\mathcal{O}$. We conclude that $W \equiv \widehat{\Gamma} \cup\left(\widehat{\gamma}^{-} \cap \mathcal{O}\right)-\gamma$ is a 1-dimensional complex subvariety in $\mathbf{P}^{n}-\gamma$.

Now by Theorem 12.8 we have $\widehat{\gamma}^{-} \cap U \subset \widehat{\Gamma}$ and therefore $\widehat{\gamma}^{-} \subset W$. However, every irreducible component of $W$ with non-empty boundary is contained in $\widehat{\gamma}$ (cf. Proposition 2.3).

13. The Projective Spectrum. In this section we introduce a projective analogue of Gelfand's representation theorem for Banach algebras. The relation of our construction to Gelfand's loosely mirrors the relation of Grothendieck's $\operatorname{Proj}\left(R_{*}\right)$ of a graded ring $R_{*}$ to the spectrum $\operatorname{Spec}(R)$ of an ordinary commutative ring $R$.

DeFinition 13.1. By a Banach graded algebra we mean a graded normed algebra

$$
A_{*}=\bigoplus_{d \geq 0} A_{d}
$$


which is a direct sum of Banach spaces. Thus the norm on $A$ is a direct sum $\|\bullet\|=$ $\|\bullet\|_{0}+\|\bullet\|_{1}+\|\bullet\|_{2}+\ldots$ where $\left(A_{d},\|\cdot\|_{d}\right)$ is complete, and $\left\|a \cdot a^{\prime}\right\|_{d+d^{\prime}} \leq\|a\|_{d}\left\|a^{\prime}\right\|_{d^{\prime}}$ for all $a \in A_{d}$ and $a^{\prime} \in A_{d^{\prime}}$, or equivalently,

$$
\|a \cdot b\| \leq\|a\|\|b\| \quad \text { for all } a, b \in A_{*} .
$$

A (degree-preserving) homomorphism of Banach graded algebras $\Psi: A_{*} \rightarrow B_{*}$ is continuous if there exists a constant $C>0$ such that $\|\Psi(a)\| \leq C^{d}\|a\|$ for all $a \in A_{d}$ and all $d \geq 0$.

EXAmple 13.2. Let $A_{*}=\mathbf{C}[t]$ be the algebra of polynomials in one variable with

$$
\|p(t)\|=\left\|\sum_{k=0}^{n} a_{k} t^{k}\right\|=\sum_{k=0}^{n}\left|a_{k}\right| .
$$

Note that the algebra automorphism $\mathbf{C}[t]$ determined by $t \mapsto c t, c \neq 0$, is continuous with a continuous inverse. This is the homogeneous coordinate ring of a projective point.

Example 13.3. Consider a compact subset $K \subset \mathbf{P}^{n}$ and let $A_{d}(K)=$ $\left.H^{0}\left(\mathbf{P}^{n}, \mathcal{O}(d)\right)\right|_{K}$ be the restriction of holomorphic sections of $\mathcal{O}_{\mathbf{P}^{n}}(d)$ to $K$. Multiplication in $A_{*}(K)$ is induced by the tensor product $\mathcal{O}(d) \otimes \mathcal{O}\left(d^{\prime}\right) \rightarrow \mathcal{O}\left(d+d^{\prime}\right)$ and the norm on $A_{d}$ is given by

$$
\|\sigma\|_{d}=\sup _{K}\|\sigma\|
$$

EXAMPLE 13.4. Let $\lambda \rightarrow X$ be a complex hermitian line bundle over a locally compact topological space $X$ and let $A_{d}(X, \lambda)=\Gamma\left(X, \lambda^{d}\right)$ denote the space of continuous sections of $\lambda^{d}$ with the sup-norm. The Banach graded algebra $A_{*}(X, \lambda)$ will sometimes be called the homogeneous coordinate ring of the polarized topological space $(X, \lambda)$.

Definition 13.5. For a Banach graded algebra $A_{*}$ we denote by

$$
H \equiv \operatorname{Hom}\left(A_{*}, \mathbf{C}[t]\right)
$$

the set of all continuous degree-preserving graded algebra homomorphisms

$$
m: A_{*} \longrightarrow \mathbf{C}[t] \text {. }
$$

By definition of continuity, for each such $m$ there is a constant $C>0$ such that

$$
|m(a)| \leq C^{d}\|a\|_{d} \quad \text { for all } a \in A_{d} \text { and all } d \geq 0 .
$$

We then set

$$
H^{\times}=H-\{\underline{0}\}
$$

where $\underline{0}$ denotes the augmentation homomorphism $\underline{0}(a)=a_{0}$. (If we write $m \in H$ as $m=\left(m_{0}, m_{1}, m_{2}, \ldots\right)$, then $\underline{0}=(1,0,0, \ldots)$.)

Definition 13.6. The projective spectrum of the graded algebra $A_{*}$ is the quotient

$$
\operatorname{Proj}\left(A_{*}\right) \equiv H^{\times} / \mathbf{C}^{\times}
$$


under the $\mathbf{C}^{\times}$-action on $H$ defined by

$$
\phi_{s}\left(\left\{m_{d}\right\}\right)=\left\{s^{d} m_{d}\right\} .
$$

Given $m \in H^{\times}$define

$$
\left\|\left|\|m \mid\| \equiv \inf \left\{C:\left|m_{d}(a)\right| \leq C^{d}\|a\|_{d} \text { for all } a \in A_{d} \text { and all } d>0\right\},\right.\right.
$$

and set

$$
S(H)=\left\{m \in H^{\times}:\|m \mid\|=1\right\} .
$$

The $\mathbf{C}^{\times}$-action on $H^{\times}$restricts to an $S^{1}$-action on $S(H)$. We introduce a topology on $\operatorname{Proj}\left(A_{*}\right)$ as follows. Embed

$$
S(H) \subset \prod_{d>0} \prod_{a \in A_{d}} D_{a}=D \quad \text { by } m \mapsto\left\{m_{d}(a)\right\}_{d, a}
$$

where $D_{a}=\{z \in \mathbf{C}:|z| \leq\|a\|\}$, and topologize $S(H)$ as a subspace of $D$ with the product topology. The circle acts continuously on $S(H)$ by standard rotation in each factor, and

$$
\operatorname{Proj}\left(A_{*}\right)=S(H) / S^{1}
$$

is given the quotient topology. Consider now the subset

$$
B(H)=\left\{m \in H^{\times}:\|m \mid\| \leq 1\right\} \subset D
$$

embedded as in (13.1) above.

Proposition 13.7. The set $B(H) \subset D$ is compact in the induced topology. The quotient $\operatorname{Proj}\left(A_{*}\right)$ is compact if and only if $\underline{0} \notin \overline{S(H)}$.

Definition 13.8. The algebra $A_{*}$ is called stable if $\underline{0} \notin \overline{S(H)}$.

Proof of Proposition 13.7. To see that $B(H)$ is closed in $D$ note that it is exactly the subset cut out by the equations:

$$
\begin{array}{cc}
z_{s a+t a^{\prime}}=s z_{a}+t z_{a^{\prime}} & \text { for } a, a^{\prime} \in A_{d}, d>0 \text { and } s, t \in \mathbf{C} \\
z_{a} z_{b}=z_{a b} & \text { for } a \in A_{d}, b \in A_{d^{\prime}}, d, d^{\prime}>0 .
\end{array}
$$

Evidently, $\operatorname{Proj}\left(A_{*}\right)=\pi(S(H))=\pi(B(H)-\{\underline{0}\})$ where $\pi: H^{\times} \rightarrow H^{\times} / \mathbf{C}^{\times}=$ $\operatorname{Proj}\left(A_{*}\right)$ is the quotient map. Hence, $\underline{0} \notin \overline{S(H)}$ implies that $\operatorname{Proj}\left(A_{*}\right)=\pi(\overline{S(H)})$ is compact. Conversely, if $\underline{0} \in \overline{S(H)}$, there is a net $Z_{\alpha}$ in $S(H)$ converging to $\underline{0}$. If $\operatorname{Proj}\left(A_{*}\right)$ were compact there would exist a subnet $Z_{\beta}$ with $\pi Z_{\beta}$ converging to some point $x \in \operatorname{Proj}\left(A_{*}\right)$. This however is impossible, since the natural continuous map $D=\prod_{a} D_{a} \rightarrow \prod_{a}[0,\|a\|]$, restricted to $S(H)$, descends to a continous map on $\operatorname{Proj}\left(A_{*}\right)$.

The concept of stability is illuminated by considering the functions $\|\bullet\|_{d}: H^{\times} \longrightarrow$ $\mathbf{R}^{+}$defined by

$$
\|m\|_{d} \equiv \sup \left\{\frac{\left|m_{d}(a)\right|}{\|a\|}: a \in A_{d}\right\} .
$$

These functions have the properties:

$$
\|m\|_{d d^{\prime}} \geq\|m\|_{d}\|m\|_{d^{\prime}}
$$




$$
\begin{aligned}
& \|\| m \|=\inf \left\{C:\|m\|_{d} \leq C^{d} \text { for all } d\right\} \\
& \|\| m\left\|=1 \quad \Leftrightarrow \quad \sup _{d}\right\| m \|_{d}=1 .
\end{aligned}
$$

Finally note that

$$
\underline{0} \in \overline{S(H)} \Leftrightarrow \exists \text { a net } m^{\alpha} \text { in } S(H) \text { s.t. } \lim _{\alpha} m_{d}^{\alpha}(a)=0 \text { for all } a \in A_{d} \text { and } d>0 .
$$

In particular,

$$
\exists \text { a net } m^{\alpha} \text { in } S(H) \text { s.t. } \lim _{\alpha}\left\|m^{\alpha}\right\|_{d}=0 \text { for all } d>0 \Rightarrow \underline{0} \in \overline{S(H)} .
$$

14. The Projective Gelfand Transform. Let $A_{*}$ be a Banach graded algebra and set $\mathcal{X}=\operatorname{Proj}\left(A_{*}\right)$. Then for each $d \geq 0$ there is a hermitian line bundle

$$
\mathcal{O}_{\mathcal{X}}(d) \longrightarrow \mathcal{X}
$$

associated to the principal $S^{1}$-bundle $S(H) \rightarrow \mathcal{X}=S(H) / S^{1}$ by the character $t^{d}$ (considered as a homomorphism $S^{1} \rightarrow S^{1}=U(1)$ ).

Let $\mathcal{A}(\mathcal{X}, d)=\Gamma\left(\mathcal{X}, \mathcal{O}_{\mathcal{X}}(d)\right)$ denote the space of continuous sections of $\mathcal{O}_{\mathcal{X}}(d)$ equipped with the sup-norm topology. Under tensor product, the direct sum

$$
\mathcal{A}_{*}(\mathcal{X}) \equiv \bigoplus_{d \geq 0} \mathcal{A}(\mathcal{X}, d)
$$

becomes a Banach graded algebra.

Observe now that in terms of continuous functions on $S(H)$ we have

$$
\mathcal{A}(\mathcal{X}, d)=\left\{S: S(H) \rightarrow \mathbf{C}: S\left(\phi_{t}(m)\right)=t^{d} S(m) \text { for all } t \in S^{1}\right\} .
$$

Hence every element $a \in A_{d}$ gives rise to an element $\widehat{a} \in \mathcal{A}(\mathcal{X}, d)$ by setting

$$
\widehat{a}(m)=m(a) .
$$

This gives an embedding

$$
A_{*} \subset \mathcal{A}_{*}(\mathcal{X}) .
$$

Proposition 14.1. For all $a \in A_{d}$ one has

$$
\|\widehat{a}\| \leq\|a\| .
$$

Thus the transformation (14.1) is a continuous injective homomorphism of $A_{*}$ into the coordinate ring of the polarized topological space $\left(\mathcal{X}, \mathcal{O}_{\mathcal{X}}(1)\right)$.

Proof. Note that

$$
\|\widehat{a}\| \equiv \sup _{[m] \in \mathcal{X}}|\widehat{a}(m)|=\sup _{m \in S(H)}|\widehat{a}(m)|=\sup _{m \in S(H)}|m(a)| \leq \sup _{m \in S(H)}\|m\|\left\|_{d}\right\| a\|\leq\| a \|
$$

since $\||m|\|=\sup _{d}\|m\|_{d}=1$. here?

Question: In the Gelfand case, one has $\|\widehat{a}\|=\lim _{n}\left\|a^{n}\right\|^{\frac{1}{n}}$. Is there an analogue 
15. Relation to the Projective Hull. Let $X \subset \mathbf{P}^{n}$ be a compact subset and $A_{*}(X)=\left.\bigoplus_{d \geq 0} H^{0}\left(\mathbf{P}^{n}, \mathcal{O}_{\mathbf{P}^{n}}(d)\right)\right|_{X}$ the algebra considered in 13.3. Set

$$
\mathcal{X} \equiv \operatorname{Proj}\left(A_{*}(X)\right) .
$$

Note the natural embedding

$$
X \hookrightarrow \mathcal{X}
$$

which assignes to $x \in X$ the equivalence class of the multiplicative functional $m_{x}$ : $A_{*}(X) \rightarrow \mathbf{C}$ obtained by choosing an indentification $\mathcal{O}_{x}(1) \cong \mathbf{C}$ and setting $m_{x}(\mathcal{P})=$ $\mathcal{P}(x)$.

Proposition 15.1. The embedding (15.1) extends to a homeomorphism

$$
\widehat{X} \cong \mathcal{X} .
$$

Proof. Let $\pi: \mathbf{C}^{n+1} \rightarrow \mathbf{P}^{n}$ denote the projection and consider the continuous mapping

$$
\pi^{-1}(\widehat{X})-\{0\} \longrightarrow H^{\times}
$$

given by $z \mapsto m_{z}$ where $m_{z}(p)=p(z)$ for homogeneous polynomials $p$. This map is $\mathbf{C}^{\times}$-equivariant, i.e., $m_{t z}=t^{d} m_{z}$ on $A_{d}$. Note that

$$
\left\|m_{z}\right\| \|=C([z])
$$

where $C=1 / \rho$ is the best constant function (cf. Prop. 5.2). Therefore the mapping (15.2) restricts to an $S^{1}$-equivariant mapping

$$
\widehat{X}_{\rho} \equiv\left\{z \in \pi^{-1}(\widehat{X}):\|z\|=\rho([z])\right\} \longrightarrow S(H),
$$

which induces a continuous mapping of the quotients

$$
\widehat{X} \longrightarrow \mathcal{X}=S(H) / S^{1} .
$$

A continuous inverse to this map is defined as follows. For $m \in H^{\times}$consider the point

$$
z=z_{m}=\left(m\left(Z_{0}\right), \ldots, m\left(Z_{n}\right)\right)
$$

where $Z_{0}, \ldots, Z_{n}$ are the standard linear coordinates in $\mathbf{C}^{n+1}$. Note that for any homogeneous polynomial $p(Z) \in \mathbf{C}\left[Z_{0}, \ldots, Z_{n}\right]$ we have

$$
m(p)=p\left(m Z_{0}, \ldots m Z_{n}\right)=p(z)=m_{z}(p) .
$$

Thus $m \mapsto z_{m}$ is a right inverse to (15.4). It is obviously also a left inverse.

COROllary 15.2. The map (15.3) is an equivariant homeomorphism. In particular, $X$ is stable iff $A_{*}(X)$ is stable.

Note that in the case considered here the Projective Gelfand transformation simply maps the "algebraic" sections of $\left.\mathcal{O}(d)\right|_{X}$ (by extension) into the continuous sections of $\left.\mathcal{O}(d)\right|_{\widehat{X}}$

16. Finitely Generated Algebras. It is a classical fact that finitely generated Banach algebras correspond to polynomially convex subsets of $\mathbf{C}^{n}$. We now show that analogously each finitely generated Banach graded algebra corresponds to a projectively convex subset of $\mathbf{P}^{n}$. 
Proposition 16.1. Let $A_{*}$ be a Banach graded algebra generated by elements $a_{0}, \ldots, a_{N} \in A_{1}$. Then the algebra homomorphism

$$
\mathbf{C}\left[Z_{0}, \ldots, Z_{n}\right] \longrightarrow A_{*}
$$

generated by $Z_{k} \mapsto a_{k}$ is a continuous surjection which induces a continuous injection

$$
\operatorname{Proj}\left(A_{*}\right) \longrightarrow \mathbf{P}^{n} \text {. }
$$

whose image is projectively convex (i.e., equal to its projective hull).

Proof. By rescaling the generators (which induces a continuous isomorphism) we may assume that $\left\|a_{k}\right\|=1$ for $k=0, \ldots, n$. Observe now that for any homogeneous polynomial $P=\sum c_{\alpha} Z^{\alpha}$,

$$
\left\|P\left(a_{0}, \ldots, a_{n}\right)\right\| \leq \sum_{\alpha}\left|c_{\alpha}\right|\left\|a_{0}\right\|^{\alpha_{0}} \ldots\left\|a_{n}\right\|^{\alpha_{n}}=\sum_{\alpha}\left|c_{\alpha}\right| \equiv\|P\|_{\infty}
$$

and $\|P\|_{\infty}$ is equivalent to the standard norm on $\mathbf{C}\left[Z_{0}, \ldots, Z_{n}\right]$ by Lemma A.1. The existence of the map (16.2) follows immediately. It is induced by the $\mathbf{C}^{\times}$-equivariant map

$$
H^{\times} \longrightarrow \mathbf{C}^{n+1}-\{0\}
$$

sending $m \mapsto\left(m\left(a_{1}\right), \ldots, m\left(a_{n}\right)\right)$. Under this map any homogeneous polynomial $P\left(Z_{0}, \ldots, Z_{n}\right)$ pulls back to $P\left(m a_{0}, \ldots, m a_{n}\right)=m\left\{P\left(a_{0}, \ldots, a_{n}\right)\right\}=\left\{P\left(a_{0}, \ldots, a_{n}\right)\right\}^{\wedge}(m)$. This is the image of $P\left(a_{0}, \ldots, a_{n}\right)$ in the homogeneous coordinate ring of $\operatorname{Proj}\left(A_{*}\right)$.

Let $\mathcal{X} \subset \mathbf{P}^{n}$ denote the image of $\operatorname{Proj}\left(A_{8}\right)$. To see that $\widehat{\mathcal{X}}=\mathcal{X}$ choose $[z] \in \widehat{\mathcal{X}}$. By definition there is a constant $C=C(z)$ such that

$$
|P(z)| \leq C^{d} \sup _{\mathcal{X}}|P|=C^{d} \sup _{m \in S(H)}|m P(a)| \leq C^{d}\|P(a)\|
$$

for all $P \in A_{d}$ and all $d$. Hence $[z] \in \mathcal{X}$.

Note 16.2. The homomorphism (16.1) is only injective when $\operatorname{Proj}\left(A_{*}\right)$ is Zariski dense. In general we get a factoring of (16.1):

$$
\mathbf{C}\left[Z_{0}, \ldots, Z_{n}\right] \stackrel{\psi}{\longrightarrow} \widetilde{A}_{*} \stackrel{\phi}{\longrightarrow} A_{*}
$$

where $\widetilde{A}_{*}$ is the quotient algebra with the quotient norm in each degree, and where $\psi$ is an algebra isomorphism which is continuous (but does not have a continuous inverse). This induces continuous injections:

$$
\operatorname{Proj}\left(A_{*}\right) \longrightarrow \operatorname{Proj}\left(\widetilde{A}_{*}\right) \longrightarrow \mathbf{P}^{n}
$$

where $\operatorname{Proj}\left(\widetilde{A}_{*}\right)$ is the Zariski hull of $\mathcal{X}=\operatorname{Proj}\left(A_{*}\right)$, i.e., the smallest algebraic subvariety containing $\mathcal{X}$.

Note 16.3. One can define a boundary for $\mathcal{X}=\operatorname{Proj} A_{*} \subset \mathbf{P}^{n}$ to be a subset $\mathcal{X}_{0} \subseteq \mathcal{X}$ with $\widehat{\mathcal{X}_{0}}=\mathcal{X}$. As opposed to the affine case, there may be no unique minimal boundary. For example if $\mathcal{X}$ is an algebraic subvariety, then any open subset, in fact any subset of positive $\omega$-capacity ( $\mathrm{cf}[\mathrm{GZ}]$ ), is a boundary. In particular boundaries can easily be disjoint.

On the other hand, for many of the examples considered here there is a unique minimal boundary. 
Note that by Theorem 12.8 , the set $\mathcal{X}-\mathcal{X}_{0}$ for any boundary $\mathcal{X}_{0}$, satisfies the Local Maximum Modulus Principle for regular functions.

17. Projective Hulls on Algebraic Manifolds. The projective hull of a subset can be defined abstractly in any projective variety. Let $X$ be a compact complex manifold provided with a hermitian line bundle $\lambda$.

Definition 17.1. The $\lambda$-hull of a compact subset $K \subset X$ is the set $\widehat{K}_{\lambda}$ of all points $x \in X$ for which there exists a constant $C=C_{x}$ such that

$$
\|\sigma(x)\| \leq C^{d} \sup _{K}\|\sigma\|
$$

for all $\sigma \in H^{0}\left(X, \mathcal{O}\left(\lambda^{d}\right)\right)$ and all $d>0$. This set is independent of the metric on $\lambda$.

Let $C_{K, \lambda}: X \rightarrow(0, \infty]$ be the best constant, defined at $x$ to be the smallest $C$ for which (17.1) holds, and set

$$
\Lambda_{K, \lambda}=\log C_{K, \lambda}
$$

This function was studied by Guedj and Zeriahi [GZ] who introduced the following. Let $\omega$ denote the curvature $(1,1)$-form of the hermitian connection on $\lambda$.

Definition 17.2. An upper semi-continuous function $v: X \rightarrow[-\infty, \infty)$ in $L^{1}(X)$ is called quasi-plurisubharmonic if

$$
d d^{c} v+\omega \geq 0
$$

The convex set of such functions will be denoted $\mathcal{P} \mathcal{S} \mathcal{H}_{\omega}(X)$

Note that the smooth functions $v \in \mathcal{P} \mathcal{S H}_{\omega}(X)$ are those with the property that the hermitian metric $e^{v}\|\cdot\|$ has non-negative curvature on $X$.

Note also that the u.s.c. function $\varphi=\frac{1}{d} \log \|\sigma\|$ with $\sigma \in H^{0}(X, \mathcal{O}(\lambda))$ is in $\mathcal{P} \mathcal{S H}_{\omega}(X)$ with $d d^{c} \varphi+\omega=\frac{1}{d} \operatorname{Div}(\sigma)$.

TheOREm 17.3. [GZ]. Let $X, \lambda$ be as above with $\lambda$ positive. Then

$$
\Lambda_{K, \lambda}(x)=\sup \left\{v(x): v \in \mathcal{P} \mathcal{S H}_{\omega}(X) \quad \text { and }\left.\quad v\right|_{K} \leq 0\right\}
$$

Furthermore, the statements of Theorem 4.3 hold with $\mathbf{P}^{n}$ replaced by $X$.

The $\lambda$-hull has the following elementary property.

LEMma 17.4. Let $\lambda \rightarrow X$ be a hermitian line bundle on a compact complex manifold. Then for any compact set $K \subset X$ and any $p \geq 1$

$$
\widehat{K}_{\lambda^{p}}=\widehat{K}_{\lambda} \quad \text { and } \quad C_{K, \lambda^{p}}=C_{K, \lambda}^{p} .
$$

Proof. It follow directly from the definitions that $\widehat{K}_{\lambda} \subseteq \widehat{K}_{\lambda^{p}}$ and $C_{K, \lambda^{p}} \leq C_{K, \lambda}^{p}$. On the other hand, suppose $x \in \widehat{K}_{\lambda^{p}}$ and $\sigma \in H^{0}\left(X, \lambda^{d}\right)$. Then

$$
\|\sigma(x)\|^{p}=\left\|\sigma^{p}(x)\right\| \leq C_{K, \lambda^{p}}^{d} \sup _{K}\left\|\sigma^{p}\right\|=C_{K, \lambda^{p}}^{d}\left(\sup _{K}\|\sigma\|\right)^{p}
$$

i.e., $\|\sigma(x)\| \leq C^{d} \sup _{K}\|\sigma\|$ where $C=\left(C_{K, \lambda^{p}}\right)^{\frac{1}{p}}$. Hence, $x \in \widehat{K}_{\lambda}$ and $C_{K, \lambda} \leq$ $\left(C_{K, \lambda^{p}}\right)^{\frac{1}{p}}$. 
We now examine the relationship of $\widehat{K}_{\lambda}$ to the projective hull of $K$ under projective embeddings related to $\lambda$. Suppose $X \subset \mathbf{P}^{N}$ is embedded by the full space of sections of $\lambda$. Then for any Borel set $K \subset X$, one has that $\widehat{K}_{X, \lambda}=\widehat{K}$, and if $\lambda$ is given the metric induced from this embedding, then $\Lambda_{K, X}=\left.\Lambda_{K, \mathbf{P}^{N}}\right|_{X}$. (Of course $\Lambda_{K} \equiv \infty$ on $\mathbf{P}^{N}-X$.) This follows from the fact that any section of $\lambda^{d}$ is the restriction of a section of $\mathcal{O}_{\mathbf{P}^{N}}(d)$. We now show that the hull remains unchanged if one embeds $X$ into projective space by any subspace of $H^{0}(X, \mathcal{O}(\lambda))$.

Proposition 17.5. Suppose $X \subset \mathbf{P}^{N}$ is an embedding given by a subspace of sections of $\lambda$. Then for any compact set $K \subset X$

$$
\widehat{K}_{\lambda}=\widehat{K} .
$$

Furthermore, is $\lambda$ is given the metric induced from this embedding, then

$$
C_{K, \lambda}=\left.C_{K}\right|_{X}
$$

where $C_{K}$ is the best constant function on $\mathbf{P}^{N}$. (Of course $C_{K} \equiv \infty$ on $\mathbf{P}^{N}-X$.)

REMARK 17.6. This result says essentially that the projective hull and the associated extremal function of a subset $K \subset \mathbf{P}^{n}$ are intrinsic to any compact submanifold $X$ containing $K$.

Proof of Proposition 17.5. This is a consequence of the following lemma. We recall that $\lambda$ is very ample if the sections of $\lambda$ give a projective embedding of $X$.

Lemma 17.7. Let $X, \lambda$ be as above and suppose $f: Y \rightarrow X$ is a holomorphic map from a compact complex manifold $Y$. Let $\mu=f^{*} \lambda$ with the induced metric. Then:

(i) $f\left(\widehat{K}_{\mu}\right) \subseteq \widehat{f(K)_{\lambda}} \quad$ and $\quad f^{*} C_{f(K), \lambda} \leq C_{K, \mu}$.

(ii) If $\lambda$ is very ample, then $\widehat{f(K)}{ }_{\lambda} \subseteq f(Y)$. then

(iii) If $\lambda$ is very ample and $f^{*}: H^{0}\left(X, \lambda^{d}\right) \rightarrow H^{0}\left(Y, \mu^{d}\right)$ is surjective for all $d$,

$$
f\left(\widehat{K}_{\mu}\right)=\widehat{f(K)_{\lambda}} \quad \text { and } \quad f^{*} C_{f(K), \lambda}=C_{K, \mu} .
$$

Proof. Suppose $y \in \widehat{K}_{\mu}$ and $\sigma \in H^{0}\left(X, \lambda^{d}\right)$. Then

$$
\|\sigma(f(y))\|=\left\|\left(f^{*} \sigma\right)(y)\right\| \leq C_{K, \mu}(y)^{d} \sup _{K}\left\|f^{*} \sigma\right\|=C_{K, \mu}(y)^{d} \sup _{f(K)}\|\sigma\| .
$$

Therefore, $f(y) \in \widehat{f(K)_{\lambda}}$ and $C_{f(K), \lambda}(f(y)) \leq C_{K, \mu}(y)$. This proves (i).

For (ii) we note that $f(K) \subset f(Y) \subset X \subset \mathbf{P}^{N}$ where the last embedding is given by the sections of $\lambda$. By (i) we have $\widehat{f(K)_{\lambda}} \subset \widehat{f(K)_{\mathcal{O}_{\mathbf{P}}(1)}}=$ the hull of $f(K)$ in $\mathbf{P}^{N}$. However, by Proposition 3.1(iii), the projective hull is contained in the Zariski hull, and so $\widehat{f(K)_{\lambda}} \subseteq f(Y)$ as claimed.

For (iii) we suppose $x \in \widehat{f(K)_{\lambda}}$, so that $\|\sigma(x)\| \leq C_{f(K), \lambda}^{d} \sup _{f(K)}\|\sigma\|$ for all $\sigma \in H^{0}\left(X, \lambda^{d}\right)$ and all $d$. Now by (ii), $x=f(y)$ for some $y \in Y$. Hence,

$$
\left\|\left(f^{*} \sigma\right)(y)\right\|=\|\sigma(f y)\| \leq C_{f(K), \lambda}(f(y))^{d} \sup _{f(K)}\|\sigma\|=C_{f(K), \lambda}(f(y))^{d} \sup _{K}\left\|f^{*} \sigma\right\|
$$


for all $\sigma \in H^{0}\left(X, \lambda^{d}\right)$ and all $d$. Therefore,

$$
\|\tau(y)\| \leq C_{f(K), \lambda}(f(y))^{d} \sup _{K}\|\tau\|
$$

for all $\tau \in H^{0}\left(Y, \mu^{d}\right)$ and all $d$ by surjectivity. Hence, $y \in \widehat{K}_{\mu}$ and so $x=f(y) \in f\left(\widehat{K}_{\mu}\right)$. Furthermore, by (17.4) we have $C_{K, \mu}(y) \leq C_{f(K), \lambda}(f(y))$. Together with part (i) this completes the proof.

18. Results for General Kähler Manifolds. In this section we derive basic results concerning hulls of sets in a general setting.

Let $X$ be a Kähler manifold with Kähler form $\omega$, and fix a compact subset $K \subset X$. Suppose $K \subset F \subset X$ with $F$ compact and define

$$
\mathcal{S} \equiv \mathcal{P} \mathcal{S H}_{\omega}(F) \equiv\left\{\varphi \in C^{\infty}(X): d d^{c} \varphi+\omega \geq 0 \text { on } F\right\},
$$

the set of smooth functions on $X$ which are quasi-plurisubharmonic on $F$.

Definition 18.1. For each $\Lambda \geq 0$ let $\widehat{K}_{F}(\Lambda)$ denote the set of all $x \in F$ such that:

$$
\varphi(x) \leq \sup _{K} \varphi+\Lambda \quad \text { for all } \varphi \in \mathcal{S} .
$$

The set

$$
\widehat{K}_{F}=\bigcup_{\Lambda \geq 0} \widehat{K}_{F}(\Lambda)
$$

will be called the $\omega$-quasi-plurisubharmonic hull of $K$ in $F$. When $X$ is compact we set $\widehat{K}(\Lambda)=\widehat{K}_{X}(\Lambda)$ and $\widehat{K}=\widehat{K}_{X}$.

Let $\mathcal{P}_{1,1}(X)$ denote the set of positive currents of bidimension $(1,1)$ with compact support on $X$, and let $\mathcal{M}_{K}$ denote the set of probability measures on $K$.

THEOREM 18.2. The following are equivalent.

$$
\text { (A) } x \in \widehat{K}_{F}(\Lambda)
$$

probablitiy

(B) There exist $T \in \mathcal{P}_{1,1}(X)$ with $M(T) \leq \Lambda$ and $\operatorname{supp}(T) \subseteq F$ and a measure $\mu \in \mathcal{M}_{K}$ such that

$$
d d^{c} T=\mu-\delta_{x} .
$$

For $\varphi \in C^{\infty}(X)$ let $L_{\varphi}$ denote the corresponding linear functional on $\mathcal{E}^{\prime}(X)$.

Lemma 18.3. The following are equivalent.

(i) $x \notin \widehat{K}_{F}(\Lambda)$

(ii) There exists $\varphi \in \mathcal{S}$ with $\sup _{K} \varphi+\Lambda<\varphi(x)$

(iii) There exists $\varphi \in \mathcal{S}$ with $\int_{K} \varphi d \mu+\Lambda<\varphi(x)$ for all $\mu \in \mathcal{M}_{K}$

(iv) There exists $\varphi \in \mathcal{S}$ such that $\mathcal{M}_{K}-\delta_{x} \subset\left\{L_{\varphi}<-\Lambda\right\}$ 
Proof. We have (i) $\Leftrightarrow$ (ii) by definition. We have (ii) $\Leftrightarrow$ (iii) because

$$
\sup _{K} \varphi=\sup _{\mu \in \mathcal{M}_{K}} \int_{K} \varphi d \mu \text {. }
$$

Note that $\mathcal{M}_{K}$ is compact, so the strict inequality in (iii) implies the strict inequality in (ii). Condition (iv) is just a restatement of (iii).

Consider the following subset of the compactly supported 0-dimensional currents on $X$ :

$$
C \equiv\left\{d d^{c} T: T \in \mathcal{P}_{1,1}(X), M(T) \leq 1 \text { and } \operatorname{supp}(T) \subseteq F\right\} .
$$

Obviously $C$ is a convex set containing the origin. It is easy to see that $C$ is compact.

Recall that for a compact convex subset $\mathcal{K}$ containing the origin in a topological vector space $V$, the polar of $\mathcal{K}$ is the set $\mathcal{K}^{0} \equiv\left\{L \in V^{*}: L \geq-1\right.$ on $\left.\mathcal{K}\right\}$.

Proposition 18.4.

$$
\mathcal{S}=C^{0} .
$$

Proof. For $u \in C$ and $\varphi \in \mathcal{S}$ we have

$$
u(\varphi)=\left(d d^{c} T\right)(\varphi)=T\left(d d^{c} \varphi+\omega\right)-T(\omega) \geq-T(\omega) \geq-1 .
$$

Hence $\mathcal{S} \subseteq C^{0}$. Conversely suppose $\varphi \in C^{0}$. Then $-1 \leq u(\varphi)=T\left(d d^{c} \varphi+\omega\right)-T(\omega)$ or

$$
0 \leq T\left(d d^{c} \varphi+\omega\right)+1-T(\omega)
$$

for all $T \in C$. Taking $T=\delta_{y} \xi$ for $y \in F$ and $\xi$ a positive simple unit $(1,1)$-vector at $y$, we have $T(\omega)=M(T)=1$ and so $\left(d d^{c} \varphi+\omega\right)(\xi) \geq 0$. This proves that $\left(d d^{c} \varphi+\omega\right)_{y} \geq 0$ for all $y \in F$.

Proposition 18.4 is equivalent to:

PROPOSITION 18.4'.

$$
\varphi \in \mathcal{S} \Leftrightarrow \Lambda C \subseteq\left\{L_{\varphi} \geq-\Lambda\right\} .
$$
$-\Lambda\}$.

Proof. $\varphi \in \mathcal{S} \Leftrightarrow \varphi \in C^{0} \Leftrightarrow C \subseteq\left\{u: L_{\varphi}(u) \geq-1\right\} \Leftrightarrow \Lambda C \subseteq\left\{u: L_{\varphi}(u) \geq\right.$

Note that

$$
\Lambda C \equiv\left\{d d^{c} T: T \in \mathcal{P}_{1,1}(X), M(T) \leq \Lambda \text { and } \operatorname{supp}(T) \subseteq F\right\} .
$$

Combining Lemma 18.3 and the Proposition 18.4' yields:

Proposition 18.5. The following are equivalent.

$$
\text { (i) } x \notin \widehat{K}_{F}(\Lambda)
$$

(v) $\exists \varphi \in C^{\infty}(X)$ with $\mathcal{M}_{K}-\delta_{x} \subset\left\{L_{\varphi}<-\Lambda\right\}$ and $\Lambda C \subset\left\{L_{\varphi} \geq-\Lambda\right\}$

Proof of Theorem 18.2. The theorem can be restated as the equivalence of:

(i) $x \notin \widehat{K}_{F}(\Lambda)$

(vi) $\mathcal{M}_{K}-\delta_{x}$ and $\Lambda C$ are disjoint.

Obviously (v) $\Rightarrow$ (vi). The Hahn-Banach Theorem states that (vi) $\Rightarrow(\mathrm{v})$. 
Suppose now that $X$ is compact and $F=X$, so that $\mathcal{S}$ is the set of all quasiplurisubharmonic functions on $X$. In this case Theorem 18.2 can be strengthened so that supp $(T) \subseteq \widehat{K}^{-}$. This is the first main result of this section.

THEOREM 18.6. Let $X$ be a compact Kähler manifold. For any compact subset $K \subset X$ the following are equivalent.

$$
\text { (A) } x \in \widehat{K}(\Lambda)
$$

(B) There exist $T \in \mathcal{P}_{1,1}$ with $M(T) \leq \Lambda$ and $\operatorname{supp}(T) \subset \widehat{K}^{-}$such that

$$
d d^{c} T=\mu-\delta_{x}
$$

where $\mu \in \mathcal{M}_{K}$.

Proof. That (B) implies (A) is already established in Theorem 18.2.

For the converse assume $x \in \widehat{K}(\Lambda)$ but the equation in (B) has no solution. Then by compactness there must exist a compact subdomain $F$ with $\widehat{K}^{-} \subset F^{0}$ such that there is no solution $T \in \mathcal{P}_{1,1}(X)$ with $M(T) \leq \Lambda$ and supp $(T) \subseteq F$. Apply Theorem 18.2 to conclude that $x \notin \widehat{K}_{F}(\Lambda)$, that is, there exists $\varphi \in C^{\infty}(X)$ which is quasi-plurisubharmonic on $F$ with $\varphi \leq 0$ on $K$ and $\varphi(x)>\Lambda$. It remains to find a function $\widetilde{\varphi}$ which is quasi-plurisubharmonic on all of $X$ and agrees with $\varphi$ on $\widehat{K}(\Lambda)$. Then $\widetilde{\varphi} \leq 0$ on $K$, and if $x \in \widehat{K}(\Lambda)$, then $\widetilde{\varphi}(x)=\varphi(x)>\Lambda$, which is a contradiction.

Proposition 18.7. Assume $X$ is a compact Kähler manifold and $\Lambda>0$. Suppose $\varphi \in C^{\infty}(X)$ is quasi-plurisubharmonic on a neighborhood of $\widehat{K}^{-}$. Then there exists a $C^{\infty}$ quasi-plurisubharmonic function $\widetilde{\varphi}$ on $X$ which agrees with $\varphi$ in a neighborhood of $\widehat{K}(\Lambda)$.

Lemma 18.8. Assume $X$ is a compact Kähler manifold and $\Lambda>0$. For each open neighborhood $U$ of $\widehat{K}^{-}$and each $N$ large, there exists a $C^{\infty}$ quasi-plurisubharmonic function $\psi$ on $X$ with $\psi>N$ on $X-U$ and $\psi<-N$ on some neighborhood of $\widehat{K}(\Lambda)$.

Proof. Note that if $\varphi \in \mathcal{P S H}_{\omega}(X)$ and $\varphi \leq 0$ on $K$, then $\varphi \leq \Lambda$ on $\widehat{K}(\Lambda)$. For each $y \in X-U$, since $y \notin \widehat{K}^{-}$, there exists $\psi \in \mathcal{P} \mathcal{S H}_{\omega}(X)$ with $\psi \leq 0$ on $K$ and $\psi(y)>2 N+\Lambda$. Set $V_{y}=\{x \in X: \psi(x)>2 N+\Lambda\}$. Extract a finite subcover $V_{1}, \ldots, V_{r}$ of $X-U$ with associated functions $\psi_{1}, \ldots, \psi_{r}$. Let $\psi=\max \left\{\psi_{1}, \ldots, \psi_{r}\right\} \in \mathcal{P S H}_{\omega}(X)$ (see [GZ, Prop. 1.3]). Then $\psi>2 N+\Lambda$ on a neighborhood of $X-U$ and $\psi \leq 0$ on $K$. Therefore $\widetilde{\psi}=\psi-N-\Lambda$ satisfies $\widetilde{\psi} \leq-N$ on $\widehat{K}(\Lambda)$ and $\widetilde{\psi}>N$ on a neighborhood of $X-U$. Finally replace $\widetilde{\psi}$ by $\psi=\widetilde{\widetilde{\psi}}-\delta$ with $\delta>0$ sufficiently small that we still have $\psi>N$ on a neighborhood of $X-U$. Then $\psi<-N$ on some neighbohood of $\widehat{K}(\Lambda)$.

Proof of Proposition 18.7. Suppose $\phi$ is quasi-plurisubharmonic on $U \supset \widehat{K}^{-}$. Now pick $N$ so that $|\varphi|<N$ on $\bar{U}$. Then $\widetilde{\varphi} \equiv \max \{\varphi, \psi\}$ satisfies:

1) $\widetilde{\varphi}=\varphi$ in a neighborhood of $\widehat{K}(\Lambda)$,

2) $\widetilde{\varphi}=\psi$ in a neighborhood of $X-U$.

Remark. The proofs of Proposition 18.7 and Lemma 18.8 only produced a continuous function since in general $\max \{\varphi, \psi\}$ is only continuous. However,

$$
\max \{\varphi, \psi\}=\lim _{n \rightarrow \infty} \frac{1}{n} \log \left(e^{n \phi}+e^{n \phi}\right)
$$


can be approximated by smooth quasi-plurisubharmonic functions (See [GZ], $\left[\mathrm{D}_{2}\right]$ ).

Theorem 18.6 can be extended to the non-compact case. On any $X$ we continue to define $\widehat{K}$ and $\widehat{K}(\Lambda)$ as in 18.1 with $F=X$.

THEOREM 18.9. Let $X$ be a non-compact Kähler manifold. Then for any compact subset $K \subset X$ with $\widehat{K} \subset \subset X$ the following are equivalent.

(A) $x \in \widehat{K}(\Lambda)$

that

(B) There exist $T \in \mathcal{P}_{1,1}(X)$ with $M(T) \leq \Lambda$ and $\operatorname{supp}(T) \subset \widehat{K}^{-}$such

$$
d d^{c} T=\mu-\delta_{x}
$$

where $\mu \in \mathcal{M}_{K}$.

Proof. Suppose $T$ is the current asserted in (B) and choose $\varphi \in \mathcal{P} \mathcal{S H}_{\omega}(X)$. Then $\int \varphi d \mu-\varphi(x)=d d^{c} T(\varphi)=T\left(d d^{c} \varphi\right)=T\left(d d^{c} \varphi+\omega\right)-T(\omega) \geq-\Lambda$, and so $x \in \widehat{K}(\Lambda)$.

Suppose now that $x \in \widehat{K}(\Lambda)$ and (B) does not hold. Then there must exist a compact subdomain $F$ with $\widehat{K}^{-} \subset F^{0}$ such that there exists no solution $T \in \mathcal{P}_{1,1}(X)$ with $M(T) \leq \Lambda$ and $\operatorname{supp}(T) \subseteq F$. Hence, by Theorem 18.2 there exists $\varphi \in$ $\mathcal{P S H}_{\omega}(\Omega)$ with $\varphi \leq 0$ on $K$ and $\varphi(x)>\Lambda$. Choose a larger compact subdomain $D$ with $F \subset \subset D^{0}$. Fix $N>\sup _{F}|\varphi|$. The argument given for Lemma 18.8 shows that there exists $\psi \in \mathcal{P} \mathcal{S H}_{\omega}(X)$ with $\psi<-N$ on a neighborhood of $\widehat{K}(\Lambda)$ and $\psi>N$ on a neighborhood of $D-F^{0}$. Define $\widetilde{\varphi} \in \mathcal{P} \mathcal{S} \mathcal{H}_{\omega}(X)$ by

$$
\widetilde{\varphi}=\left\{\begin{array}{cl}
\max \{\varphi, \psi\} & \text { on } D \\
\psi & \text { on } X-D
\end{array}\right.
$$

and note that $\widetilde{\varphi}=\varphi$ in a neighborhood of $\widehat{K}(\Lambda)$. However, $\widetilde{\varphi} \leq 0$ on $K$ and $\widetilde{\varphi}(x)>\Lambda$, so $x \notin \widehat{K}(\Lambda)$, a contradiction.

REMARK 18.10. The analogues of Corollaries 11.3 and 11.4, and Theorems 12.1 and 12.3 hold in this context. Moreover, the following analogue of Theorem 12.8 holds.

Fix $K^{\text {compact }} \subset X$ and $U^{\text {open }} \subset \subset \Omega^{\text {open }} \subset X$ where $\Omega$ is analytically equivalent to a Runge domain in $\mathbf{C}^{n}$. Then

$$
\widehat{K}^{-} \cap U \subseteq \Omega \text {-HolomorphicHull }\left\{\left(\widehat{K}^{-} \cap \partial U\right) \cup(K \cap U)\right\}
$$

with equality if $C_{K}$ is bounded on $\widehat{K} \cap \bar{U}$.

REMARK 18.11. Much of the discussion of section 4 holds in this general context. The capacity of Dinh-Sibony [DiS] was introduced for any Kähler manifold $X$ and the Theorem 4.3 of Guedj-Zeriahi holds there. Furthermore, Dinh-Sibony [DiS] proved that any analytic subvariety $Z \subset X$ is always globally $\omega$-pluripolar. Hence, if $K \subset Z$, then $\widehat{K} \subset Z$, and so $\widehat{K}$ is contained in the "analytic hull" of $K$, that is, the intersection of all subvarieties of $X$ which contain $K$.

Appendix A. Norms on $A_{*}\left(\mathbf{P}^{n}\right)$. From one point of view this paper is simply concerned with the study of equivalence classes of norms on the graded algebra $\mathbf{C}\left[Z_{0}, \ldots, Z_{n}\right]$. Two norms $\|\bullet\|$ and $\|\bullet\|^{\prime}$ are equivalent if there exists a constant 
$C>0$ such that $\frac{1}{C^{d}}\|a\| \leq\|a\|^{\prime} \leq C^{d}\|a\|$ for all $a \in A_{d}$ or equivalently, if the identity $\operatorname{map}\left(A_{*},\|\bullet\|\right) \rightarrow\left(A_{*},\|\bullet\|^{\prime}\right)$ is continuous in both directions. There are many norms equivalent to the standard one given by (2.7). We examine some of them here.

Let $\Omega \subset \mathbf{C}^{n}$ be a closed bounded convex set and define

$$
\|P\|_{\Omega} \equiv \sup _{\Omega}|P|
$$

for $P \in \mathbf{C}\left[Z_{0}, \ldots, Z_{n}\right]_{d}$. Then obviously

$$
\begin{aligned}
& \text { (i) } \Omega_{1} \subset \Omega_{2} \Rightarrow\|P\|_{\Omega_{1}} \leq\|P\|_{\Omega_{2}} \\
& \text { (ii) }\|P\|_{t \Omega}=|t|^{d}\|P\|_{\Omega}
\end{aligned}
$$

for all $P \in \mathbf{C}\left[Z_{0}, \ldots, Z_{n}\right]_{d}$. It follows easily that all these norms are equivalent.

Another interesting norm on $\mathbf{C}\left[Z_{0}, \ldots, Z_{n}\right]$ is defined on $P(Z)=\sum_{|\alpha|=d} c_{\alpha} Z^{\alpha}$ by

$$
\|P\|_{\infty} \equiv \sum_{|\alpha|=d}\left|c_{\alpha}\right|
$$

Lemma A.1. The norms $\|\bullet\|_{\infty}$ and $\|\bullet\|_{\Omega}$ are equivalent. Note that

Proof. We shall work with the polydisk $\Omega=\left\{Z \in \mathbf{C}^{n+1}:\left|Z_{k}\right| \leq 1\right.$ for all $\left.k\right\}$.

$$
\begin{aligned}
\|P\|_{\Omega} & =\sup _{\left|Z_{0}\right|=\cdots=\left|Z_{n}\right|=1}|P(Z)|=\sup _{\left|Z_{0}\right|=\cdots=\left|Z_{n}\right|=1}\left|\sum_{|\alpha|=d} c_{\alpha} Z^{\alpha}\right| \\
& \leq \sup _{\left|Z_{0}\right|=\cdots=\left|Z_{n}\right|=1} \sum_{|\alpha|=d}\left|c_{\alpha}\right|\left|Z^{\alpha}\right|=\sum_{|\alpha|=d}\left|c_{\alpha}\right|=\|P\|_{\infty} .
\end{aligned}
$$

For the converse assume inductively that

$$
\|P\|_{\infty} \leq C^{d}\|P\|_{\Omega}
$$

for all $P \in \mathbf{C}[Z]_{d}$, and all $d \leq N-1$ where $C=(n+1) 4^{n+1}$. Fix $P=\sum_{\alpha} c_{\alpha} Z^{\alpha} \in$ $\mathbf{C}[Z]_{N}$ and note that

$$
\frac{\partial P}{\partial Z_{k}}=\sum_{|\alpha|=N} \alpha_{k} c_{\alpha} Z^{\alpha-\epsilon_{k}} \in \mathbf{C}[Z]_{N-1}
$$

Hence by induction

$$
\sum_{\alpha} \alpha_{k}\left|c_{\alpha}\right| \leq C^{N-1}\left\|\frac{\partial P}{\partial Z_{k}}\right\|_{\Omega}
$$

Now for $Z \in \Omega$,

$$
\frac{\partial P}{\partial Z_{k}}(Z)=\left(\frac{1}{2 \pi i}\right)^{n+1} \int_{\left|\zeta_{0}\right|=2} \ldots \int_{\left|\zeta_{n}\right|=2} \frac{P(\zeta) d \zeta_{0} \ldots d \zeta_{n}}{\left(\zeta_{k}-z_{k}\right) \prod_{j=0}^{n}\left(\zeta_{j}-z_{j}\right)}
$$


from which it follows that

$$
\begin{aligned}
\left\|\frac{\partial P}{\partial Z_{k}}\right\|_{\Omega} & \leq\left(\frac{1}{2 \pi}\right)^{n+1} \int_{0}^{2 \pi} \cdots \int_{0}^{2 \pi}\left|P\left(2 e^{i \theta_{0}}, \ldots, 2 e^{i \theta_{n}}\right)\right| 2^{n+1} d \theta_{0} \ldots d \theta_{n} \\
& \leq 2^{n+1} \sup _{\left|\zeta_{0}\right|=\cdots=\left|\zeta_{n}\right|=2}|P(\zeta)| \leq 4^{n+1} \sup _{\left|\zeta_{0}\right|=\cdots=\left|\zeta_{n}\right|=1}|P(\zeta)|=4^{n+1}|| P \|_{\Omega}
\end{aligned}
$$

Therefore we have

$$
\begin{aligned}
\|P\|_{\infty} & =\sum_{\alpha}\left|c_{\alpha}\right| \leq \sum_{k=0}^{n} \alpha_{k}\left|c_{\alpha}\right|=\sum_{k=0}^{n}\left\|\frac{\partial P}{\partial Z_{k}}\right\|_{\infty} \\
& \leq C^{N-1} \sum_{k=0}^{n}\left\|\frac{\partial P}{\partial Z_{k}}\right\|_{\Omega} \leq C^{N-1}(n+1) 4^{n+1}\|P\|_{\Omega}=C^{N}\|P\|_{\Omega}
\end{aligned}
$$

as desired.

\section{REFERENCES}

[A 1 H. Alexander, Polynomial approximation and hulls in sets of finite linear measure in $\mathbf{C}^{n}$, Amer. J. Math., 93 (1971), pp. 65-74.

$\left[\mathrm{A}_{2}\right]$ H. Alexander, Projective capacity., in Recent developments in several complex variables (Proc. Conf., Princeton Univ., 1979), Ann. of Math. Studies No. 100, Princeton Univ. Press, Princeton, N.J., 1981, pp. 3-27.

[AW $\left.{ }_{1}\right]$ H. Alexander And J. Wermer, Several Complex Variables and Banach Algebras, SpringerVerlag, New York, 1998.

$\left[\mathrm{AW}_{2}\right]$ H. Alexander And J. Wermer, Linking numbers and boundaries of varieties, Ann. of Math., 151 (2000), pp. 125-150.

[BT] E. Bedford AND B. A. TAYlor, A new capacity for plurisubharmonic functions, Acta Math., 149 (1982), pp. 1-40.

[ClP] D. Coman, N. Levenberg And E. Poletsky, Quasianalyticity and pluripolarity, J. Amer. Math. Soc., 18:2 (2005), pp. 239-252. ArXiv:math.CV/0402381.

[D $\mathrm{D}_{1}$ J.-P. Demailly, Estimations $L^{2}$ pour l'opérateur $\bar{\partial}$ d'un fibré vectoriel holomorphe semipositif au-dessus d'une variété kählérienne complète, Ann. Sci. E. N. S. (4), 15 (1982), pp. 457-511.

$\left[\mathrm{D}_{2}\right]$ J.-P. Demailly, Regularization of closed positive currents and intersection theory, J. Algebraic Geom., 1 (1992), pp. 361-409.

[DPS] J.-P. Demailly, T. Peternell And M Schneider, Pseudo-effective line bundles on compact Káhler manifolds, Internat. J. Math., 12 (2001), pp. 689-741.

[DF] K. Diederich and J. E. Fornaess, A smooth curve in $\mathbf{C}^{2}$ which is not a pluripolar set, Duke Math. J., 49 (1982), pp. 931-936.

[DL] T.-C. Dinh and M. Lawrence, Polynomial hulls and positive currents, Ann. Fac. Sci de Toulouse, 12 (2003), pp. 317-334.

[DiS] T.-C. Dinh And N. Sibony, Distribution des valeurs des transformations meromorphes et applications, Preprint ArXiv:math. DS/0306095 (2003).

[Do] P. Dolbeault, On holomorphic chains with given boundary in $\mathbf{C P}^{n}$, Springer Lecture Notes, no. 1089, (1983), pp. 1135-1140.

[DH 1 P. Dolbeault and G. Henkin, Surfaces de Riemann de bord donné dans $\mathbf{C P}^{n}$, in Contributions to complex analysis and analytic geometry, pp. 163-187 in Aspects of Math., E26, Vieweg, Branuschweig, 1994.

[DH $\left.\mathrm{DH}_{2}\right]$ P. Dolbeault and G. Henkin, Chaines holomorphes de bord donné dans $\mathbf{C P}^{n}$, Bull. Soc. Math. de France, 125 (1997), pp. 383-445. 
[DS] J. Duval and N. Sibony, Polynomial convexity, rational convexity and currents, Duke Math. J., 79 (1995), pp. 487-513.

[Fa $\left.{ }_{1}\right]$ B. FABRÉ, Sur l'intersection d'une surface de Riemann avec des hypersurfaces algébriques, C. R. Acad. Sci. Paris, 322 Série I (1996), pp. 371-376.

$\left[\mathrm{Fa}_{2}\right]$ B. FABRÉ, On the support on complete intersection 0-cycles, The Journal of Geometric Analysis, 12 (2002), pp. 601-614.

[F] H. Federer, Geometric Measure Theory, Springer-Verlag, New York, 1969.

[G] I. M. Gelfand, Normierte Ringe, Math. Sb. (N.S.), 9 (51) (1941), pp. 3-24.

[GZ] V. Guedu And A. Zeriahi, Intrinsic capacities on compact Kähler manifolds, Preprint Univ. de Toulouse (2003), ArXiv:math.CV/0401302.

[HL $\left.{ }_{1}\right]$ F.R. Harvey and H.B. Lawson, JR., On boundaries of complex analytic varieties, I, Ann. of Math., 102 (1975), pp. 223-290.

[HL $\left.\mathrm{H}_{2}\right]$ F.R. HARVEy AND H.B. LAWSON, JR., On boundaries of complex analytic varieties, II, Ann. of Math., 106 (1977), pp. 213-238.

[HL $\left.\mathrm{H}_{3}\right]$ F.R. Harvey And H.B. Lawson, JR., Boundaries of varieties in projective manifolds, Jour. of Geom. Analysis, 14 no. 4 (2005), ArXiv:math.CV/0512490.

[HL $\left.{ }_{4}\right]$ F.R. Harvey And H.B. Lawson, JR., Projective linking and boundaries of positive holomorphic chains in projective manifolds, Stony Brook preprint (2005), ArXiv:math. $\mathrm{CV} / 0512379$.

[Hi] E. Hille, Analytic Function Theory, Vol II, Ginn and Co., Boston, 1962.

[Ho] L. Hörmander, An Introduction to Complex Analysis in Several Variables, Van Nostrand, Princeton, N. J., 1966.

[LMP] N. Levenberg, G. Martin and E. A. Poletsky, Analytic disks and pluripolar sets, Indiana Univ. Math. J., 41 (1992), pp. 515-532.

[R] M. Rosenlicht, Generalized Jacobian Varieties, Ann. of Math., 59 (1954), pp. 505-530.

[S] A. Sadullaev, An estimate for polynomials on analytic sets, Math. USSR Izvestia, 20 No. 3 (1983), pp. 493-502.

[Sib] N. SibONY, multi-dimensional analytic structure in the spectrum of a uniform algebra, in Spaces of analytic functions. (Seminar on Functional Analysis and Function Theory, Kristiansand, 1975), Springer Lect. Notes in Math. No. 512, 1976, pp. 139-165.

[SW] N. Sibony and P.-M. Wong, Some results on global analytic sets, Springer Séminaire Pierre Lelong-Henri Skoda (Analyse). Années 1978-79. pp. 221-237, Lect. Notes in Math., 822 (1980).

[Si] J. SicIaK, On some extremal functions and their applications in the theory of analytic functions in several variables, Trans. Amer. Math. Soc., 105 (1962), pp. 322-357.

[Wie] J. Wiegerinck, Pluripolar sets: hulls and completeness, Actes des Rencontres d'Analyse Complexe (Poitiers-Futuroscope, 1999), Atlantique, Poitiers, 2002, pp. 209-219.

[Wo] J. Wermer, Banach Algebras and Several Complex Variables, Springer-Verlag, New York, 1976.

[W1] J. Wermer, The hull of a curve in $\mathbf{C}^{n}$, Ann. of Math., 68 (1958), pp. 550-561.

$\left[\mathrm{W}_{2}\right]$ J. WeRmer, The argument principle and boundaries of analytic varieties, Operator Theory: Advances and Applications, 127 (2001), pp. 639-659.

[Z] A. ZeRIAHI, A criterion of algebraicity for Lelong classes and analytic sets, Acta Math., 184 (2000), pp. 113-143. 